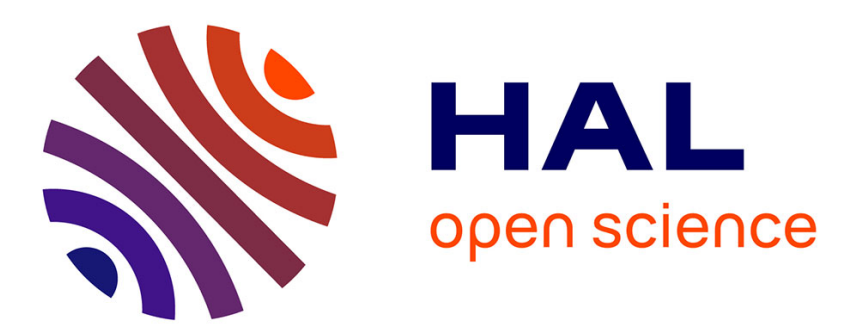

\title{
A comprehensive theoretical comparison of proton imaging set-ups in terms of spatial resolution
}

\author{
N. Krah, Feriel Khellaf, Jean Michel Létang, S. Rit, I. Rinaldi
}

\section{To cite this version:}

N. Krah, Feriel Khellaf, Jean Michel Létang, S. Rit, I. Rinaldi. A comprehensive theoretical comparison of proton imaging set-ups in terms of spatial resolution. Physics in Medicine and Biology, 2018, 63 (13), 10.1088/1361-6560/aaca1f . hal-01909706

\section{HAL Id: hal-01909706 https://hal.science/hal-01909706}

Submitted on 26 Nov 2019

HAL is a multi-disciplinary open access archive for the deposit and dissemination of scientific research documents, whether they are published or not. The documents may come from teaching and research institutions in France or abroad, or from public or private research centers.
L'archive ouverte pluridisciplinaire HAL, est destinée au dépôt et à la diffusion de documents scientifiques de niveau recherche, publiés ou non, émanant des établissements d'enseignement et de recherche français ou étrangers, des laboratoires publics ou privés. 


\title{
A comprehensive theoretical comparison of proton imag- ing set-ups in terms of spatial resolution
}

\author{
N. Krah ${ }^{1,2}$, F. Khellaf ${ }^{1}$, JM. Létang ${ }^{1}$, S. Rit ${ }^{1}$, I. Rinaldi ${ }^{2}$ \\ ${ }^{1}$ Lyon University, INSA-Lyon, University Lyon1, UJM-Saint Etienne, CNRS, Inserm, CREATIS UMR5220, U1206, France \\ ${ }^{2} \mathrm{CNRS} / \mathrm{IN} 2 \mathrm{P} 3$ and Lyon 1 University, UMR 5822, Villeurbanne, France \\ nils.krah@creatis.insa-lyon.fr
}

\begin{abstract}
We present a comprehensive analytical comparison of four types of proton imaging set-ups and, to this end, develop a mathematical framework to calculate the width of the uncertainty envelope around the most likely proton path depending on set-up geometry, detector properties, and proton beam parameters. As a figure of merit for the spatial resolution achievable with each set-up, we use the frequency $f_{10 \%}$ at which the modular transfer function of a density step decreases below $10 \%$. We verify the analytical results with Monte Carlo simulations.

We find that set-ups which track the angle and position of individual protons in front of and behind the phantom would yield an average spatial resolution of $0.3-0.35 \mathrm{lp} / \mathrm{mm}$ assuming realistic geometric parameters (i.e., $30-40 \mathrm{~cm}$ distance between detector and phantom, 15$20 \mathrm{~cm}$ phantom thickness). For set-ups combining pencil beam scanning with either a position sensitive detector, e.g., an X-ray flat panel, or with a position insensitive detector, e.g., a range telescope, we find an average spatial resolution of about $0.1 \mathrm{lp} / \mathrm{mm}$ for an $8 \mathrm{~mm}$ FWHM beam spot size. The pixel information improves the spatial resolution by less than $10 \%$. In both set-up types, performance can be significantly improved by reducing the pencil beam size down to $2 \mathrm{~mm}$ FWHM. In this case, the achievable spatial resolution reaches about $0.25 \mathrm{lp} / \mathrm{mm}$. Our results show that imaging set-ups combining double scattering with a pixel detector can provide sufficient spatial resolution only under very stringent conditions and are not ideally suited for computed tomography applications. We further propose a region-ofinterest method for set-ups with a pixel detector to filter out protons which have undergone nuclear reactions and discuss the impact of tracker detector uncertainties on the most likely path.
\end{abstract}

This is a peer-reviewed, un-copyedited version of an article accepted for publication/published in Physics in Medicine and Biology. IOP Publishing Ltd is not responsible for any errors or omissions in this version of the manuscript or any version derived from it. The Version of Record is available online at https://doi.org/10.1088/1361-6560/aaca1f 


\section{INTRODUCTION}

Proton therapy is a rapidly growing technique for tumour radiation therapy. The main advantage of proton therapy over conventional radiation therapy (performed with X-rays or electrons) is the ability of delivering no dose beyond the tumour and a much lower dose in front of the tumour (Paganetti 2012b). This feature arises from the proton depth-dose distribution where the maximum dose is deposited at the end of the proton range, in the so called Bragg-peak region, contrarily to photons which deposit their maximum dose on or near the patient surface. Range uncertainties represent an important caveat for the exploitation of the full potential of proton therapy treatments. To reduce them and to produce more conformal treatment plans, proton imaging can serve as complementary and/or alternative imaging modality for proton beam therapy (Schneider et al. 2005, Schulte \& Penfold 2012, Arbor et al. 2015). The main reason is the following: In a regular proton treatment work-flow, the patient undergoes an $\mathrm{X}$-ray planning computed tomography $(\mathrm{CT})$, on which the tumour is contoured and the plan defined. The patient anatomical information from the CT needs to be converted from X-ray Hounsfield Unit (HU), which is a measure of photon attenuation, into proton relative stopping power (RSP), from which one can obtain the proton range in the patient. Such a CT image, expressed in RSP, serves as anatomical map needed by the treatment planning system (TPS) to calculate the treatment plan. The HU-RSP conversion represents an important source of range uncertainties in proton therapy nowadays, amounting to about $2 \%$ of the proton range (Paganetti 2012b). Contrary to an X-ray CT, proton imaging directly measures the water equivalent thickness (WET), equivalent to the RSP integrated along aligned voxels, without the need for any conversion and without the inherent range uncertainties (Schulte \& Penfold 2012). In spite of its potential advantage as a direct probe of WET/RSP, proton imaging is not yet used in clinics. The main two reasons are the inferior spatial resolution of proton CT compared to X-ray CT and the necessary integration of a proton imaging set-up in the treatment facility.

In the past decades, different types of proton imaging set-ups have been proposed, developed and investigated (Parodi 2014, Poludniowski et al. 2015, Johnson 2018). All have in common that protons are shot through the patient/phantom at high enough energy so that they emerge on the other side where they are captured in a suitable detector device. The WET of the traversed material is typically determined either from the residual energy or the residual range of the protons. Some set-ups collect information about single protons individually. In particular, pairs of tracker devices located in front of and behind the patient register the position and angle of each proton. Another group of imaging devices measure entire ensembles of protons, either delivered by active pencil beam scanning (PBS) or by passive double scattering. They typically employ either a range telescope or a single plane imager panel (otherwise employed in X-ray imaging) in combination with beam energy modulation to measure the WET of the phantom or tissue material. We will provide the relevant details of each set-up in Sec. 2.1.

Protons undergo random multiple Coulomb scattering (MCS) events within the traversed material which cause their trajectories to follow a stochastic "zig-zag" shape (Gottschalk et al. 1993). This physical effect limits the spatial resolution of proton radiographic images and consequently also of the reconstructed volumetric images. On the other hand, a certain amount of spatial resolution is necessary in order for proton imaging to be useful as a complementary imaging modality in a proton therapy facility. How much this ought to be depends on the specific application (range verification, patient positioning, stopping power calibration, treatment planning CT) and deserves a systematic study on its own. In a scenario in which proton CT is used as input for a TPS instead of the conventional X-ray CT, the Nyquist frequency associated with the voxel grid provides at least an order of magnitude. For a $2 \mathrm{~mm}$ voxel spacing, representative of what is typically used in commercial TPS, the Nyquist frequency is $0.25 \mathrm{lp} / \mathrm{mm}$. A proton CT image with a lower spatial resolution than this number will potentially be insufficient for accurate treatment planning on the desired dose grid. On the other hand, a much higher spatial resolution will probably be unnecessary because the discretisation on the voxel 

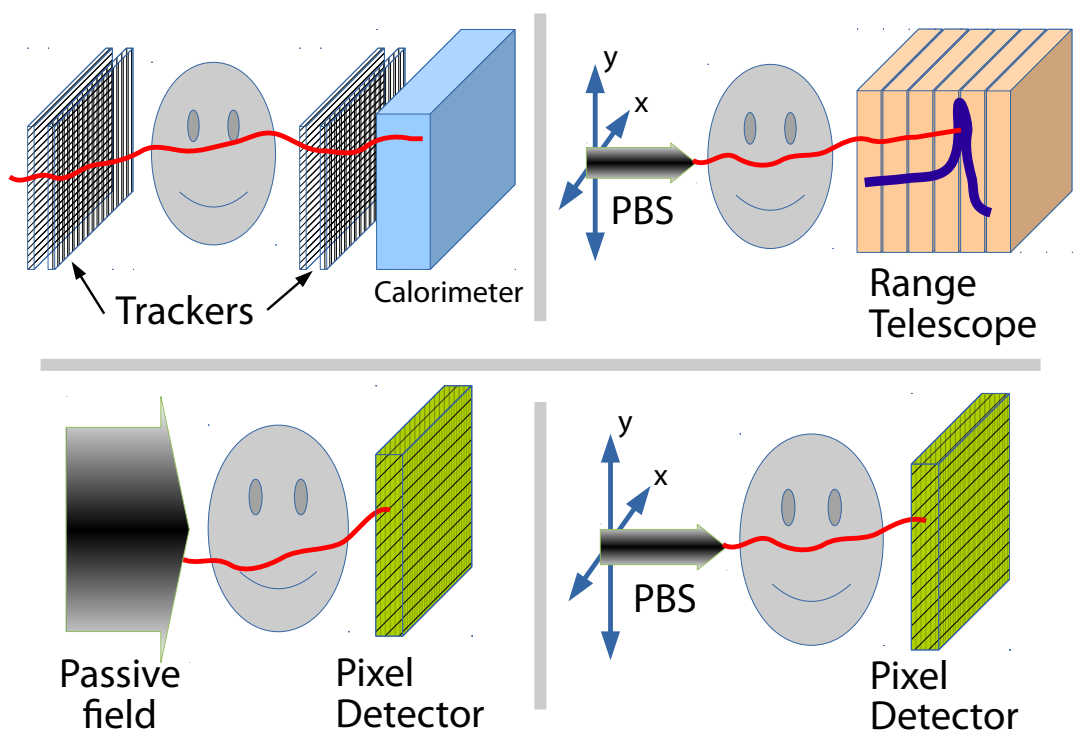

Figure 1: Schematic illustration of the four types of proton imaging set-ups compared in this work.

grid would anyhow be unable to appreciate the higher spatial frequencies.

For a specific category of set-ups, namely those measuring the protons' entry and exit coordinates, the impact of parameters such as the distance between the phantom and the trackers or the internal tracker construction on the spatial resolution has been studied (Penfold et al. 2011, Schneider et al. 2012, Bopp et al. 2014). In this work, we contribute a comprehensive comparison of the most common types of proposed set-ups in terms of the characteristic spatial resolution they are able to achieve.

\section{MATERIAL AND METHODS}

\section{$2.1 \quad$ Set-ups}

We considered four types of proton imaging set-ups in this work which have all been investigated by several groups in the past or are currently being developed. They are illustrated schematically in Fig. 1. To acquire three-dimensional tomographic images, either the patient needs to be rotated around its vertical axis or the beam nozzle together with the imaging equipment needs to be rotated around the patient so that projections can be recorded under a range of angles and used as input data for a suitable tomographic reconstruction algorithm. It is not the purpose of this work to compare the performance of these algorithms.

In the following, we briefly describe the set-ups and underline the characteristic aspects which have an impact on the spatial resolution.

Single tracking (Upper left panel in Fig. 1) In what is often referred to as "single tracking", the set-up includes pairs of tracker devices placed in front of the phantom (patient) and behind it (Schulte et al. 2004, Penfold et al. 2011, Scaringella et al. 2014, Taylor et al. 2015, Taylor et al. 2016, Civinini et al. 2013). They measure the position and angle of a proton before entering and after exiting the phantom. An additional detector, typically a calorimeter, measures the residual energy of the protons from which the water equivalent thickness of the phantom can be deduced (Sipala et al. 2015). Such a set-up requires a sufficiently high acquisition rate and/or a sufficiently low particle fluence to be able to register single proton events. A slightly 
simpler version of this set-up measures only the protons' position and not their angle (Pemler et al. 1999, Schneider et al. 2004, Shinoda et al. 2006, Schneider et al. 2012). In this work, we mainly concentrate on single tracking set-ups which do measure the entry and exit angle.

Passive field + pixel detector (Lower left panel in Fig. 1) In a treatment facility with passive double scattering beam delivery, it has been proposed to irradiate the patient with an extended field and capture the protons with a position sensitive single plane detector placed behind the patient (Zygmanski et al. 2000, Lu 2008, Muraishi et al. 2009, Seco \& Depauw 2011, Testa et al. 2013). The initial beam energy is modulated, e.g., with a spinning wheel with decreasing material thickness, while each detector pixel records the signal over time. The WET of the patient is estimated pixel per pixel from the shape of the so-obtained dose rate functions (Jee et al. 2017, Zhang et al. 2018). We will refer to this type of set-up with the abbreviated term "passive + pixel".

PBS + pixel detector (Lower right panel in Fig. 1) A similar set-up can be used in combination with pencil beam scanning (PBS). Again, the initial beam energy must be modulated, either directly in case of a synchrotron accelerator or through a range modulator device in case of a cyclotron accelerator (Bentefour et al. 2016). (Telsemeyer et al. 2012) used such a set-up in combination with carbon ion beams, but the principles are equivalent. The WET is determined essentially by identifying the beam energy for which the protons range out in the single plane detector. Contrary to the passive field set-up, the protons are known to have entered the patient in a relatively small region $\left(\approx 50-100 \mathrm{~mm}^{2}\right)$ around the centre of the pencil beam. Therefore, geometrical information is available to the tomographic reconstruction algorithm, both, by the pencil beam position and the pixel location. We call this type of set-up "PBS + pixel detector".

PBS + range telescope (Upper right panel in Fig. 1) The fourth type of set-up considered in this work combines pencil beam scanning with a detector which is not position sensitive. When using a range telescope, typically realized as multi layer ionisation chamber (Rinaldi et al. 2013, Rinaldi et al. 2014, Farace et al. 2016), the WET can be estimated from the measured integrated depth dose profiles, essentially from the Bragg peak position (Krah et al. 2015). Because range information is obtained from the integral signal over the entire ionisation chamber planes, the detector does not provide geometrical information. A two dimensional image is constructed based on the known pencil beam spot position, so that each spot corresponds to one image pixel. Alternatively, a calorimeter can be used instead of the range telescope to measure the protons residual energy (Rescigno et al. 2015). We use the term "PBS + range telescope" to collectively refer to this set-up category.

The above descriptions are to summarise the most important aspects of the four imaging set-ups and many technical details could certainly be added to each of them. We wish to underline that the purpose of this work is to compare types of imaging systems based on the characteristic properties rather than to make statements about specific implementations of these systems.

\subsection{Recapitulation: Estimation of the Most Likely Path}

The spatial resolution of proton images (radiographic - 2D and tomographic - 3D) is limited because of a combination of factors: the random scattering of protons within the patient as well as parameters characteristic of the imaging set-up and the proton beam. The purpose of this work is to systematically compare the four types of set-ups under the aspect of spatial image resolution. The mathematical framework which is typically used to describe the effect of multiple Coulomb scattering (MCS) on proton trajectories in the context of proton CT is a good starting point. We therefore recapitulate the central aspects for coherence and better legibility. 


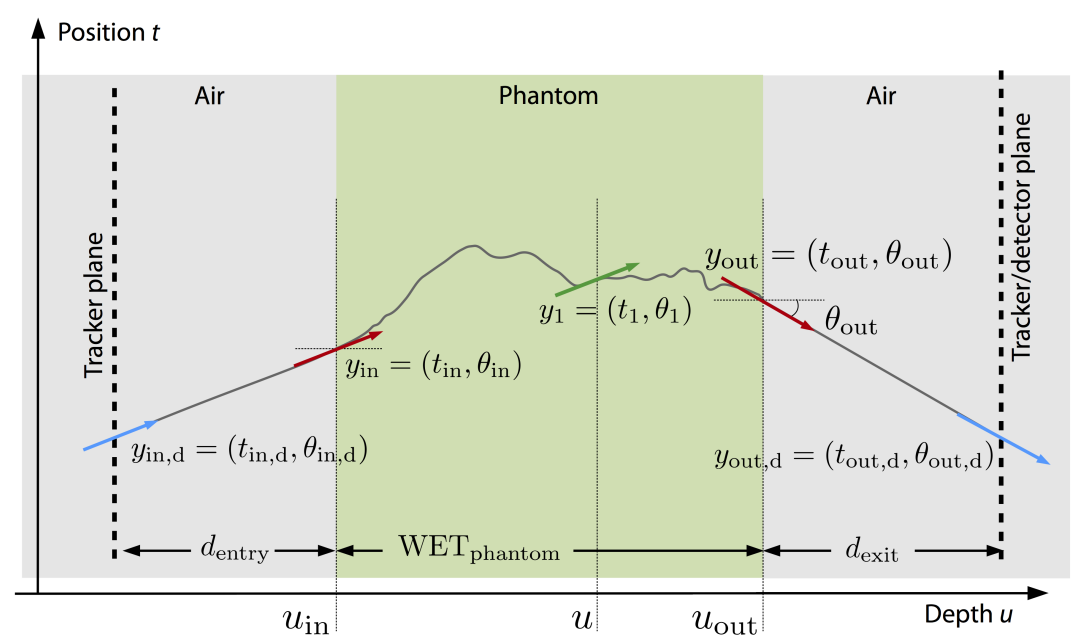

Figure 2: Sketch to illustrate the geometrical definitions used in the mathematical derivations. Coloured arrows indicate the position and propagation direction of a proton. The solid "zigzag" line is to exemplify a single proton trajectory. The dashed vertical lines summarise pairs of tracker detectors each.

Several authors have reported mathematical formalisms (Williams 2004, Schulte et al. 2008, Erdelyi 2009, Collins-Fekete et al. 2016) to estimate the most likely proton path when using single tracking set-ups. As a by-product, the mathematical equations also allow us to quantify the size of the uncertainty envelope around the most likely path. It is this latter quantity on which we mainly concentrate in this paper.

The existing formalisms make the simplifying assumption that the phantom is homogeneous and made of water and so do we. Furthermore, scattering in the two directions perpendicular to the beam is treated independently. In what follows, we refer to one plane only. We denote the proton position in the transversal direction with $t$, its propagation angle with $\theta$, and the combined vector as $y=(t, \theta)$. We have summarized all variables graphically in Fig. 2. Part of the illustration will be relevant only in Sec. 2.4 .

The rationale behind the existing MLP formalisms is as follows: A proton entering the phantom at $y_{\text {in }}$ undergoes a large number of small scattering events. In some given depth $u$, it therefore passes a random point $y_{1}=\left(t_{1}, \theta_{1}\right)$ and exits the phantom at $u_{\text {out }}$ at some random $y_{2}=\left(t_{2}, \theta_{2}\right)$. One defines the joint likelihood

$$
L\left(y_{1}, y_{2} \mid y_{\text {in }}\right)
$$

that the proton passes at $y_{1}$ and $y_{2}$, given the entrance parameter $y_{\text {in }}$. Mathematically, this is a function of two variables which both result from the same random process: the MCS. One of the variables is fixed to the value $y_{\text {out }}$ measured by a suitable tracking device behind the phantom, so that

$$
L\left(y_{1}, y_{2}=y_{\text {out }} \mid y_{\text {in }}\right)
$$

becomes a function of $y_{1}$ only.

Using the chain rule for joint probabilities, Eq. 2 may be expanded in two different ways:

$$
\begin{aligned}
L\left(y_{1}, y_{2}=y_{\text {out }} \mid y_{\text {in }}\right) & =L\left(y_{1} \mid y_{2}=y_{\text {out }} ; y_{\text {in }}\right) \times L\left(y_{2}=y_{\text {out }} \mid y_{\text {in }}\right) \\
& =L\left(y_{2}=y_{\text {out }} \mid y_{1} ; y_{\text {in }}\right) \times L\left(y_{1} \mid y_{\text {in }}\right)
\end{aligned}
$$

We remark that $y_{\text {in }}$ is not a variable in terms of the random scattering process, but rather an input parameter, which is why we have used a semicolon to separate it from the conditional 
variable. The chain rule for joint probabilities is sometimes called "Bayes' theorem" in statistics textbooks which may have led other authors to refer to "Bayesian statistics" when deriving the MLP.

Equation 3 would be used for example in a Monte Carlo (MC) simulation, in which many protons depart from $y_{\text {in }}$, but only those with a certain $y_{2}=y_{\text {out }}$ are selected for analysing the distribution of $t_{1}$ and $\theta_{1}$ in some depth $u$. The term $L\left(y_{2}=y_{\text {out }} \mid y_{\text {in }}\right)$ is actually only a constant factor in this case in that it does not depend on $y_{1}$.

Equation 4, on the other hand, lends itself to a physics motivated statistical model and indeed underlies the MLP formalisms. To this end, the two factors are interpreted as likelihood of a proton to be scattered from $y_{\text {in }}$ to $y_{1}$ and from $y_{1}$ to $y_{2}=y_{\text {out }}$, respectively. Equation 4 becomes

$$
L\left(y_{1}, y_{2}=y_{\text {out }} \mid y_{\text {in }}\right)=L_{\text {scat }}\left(y_{\text {in }} \rightarrow y_{1}\right) \times L_{\text {scat }}\left(y_{1} \rightarrow y_{2}=y_{\text {out }} \mid y_{\text {in }}\right) .
$$

The condition $y_{\text {in }}$ in the second term is actually superfluous and we will henceforth drop it for simplicity. The two factors in the above equation are modelled as Gaussian likelihood functions (Schulte et al. 2008):

$$
\begin{aligned}
L_{\text {scat }}\left(y_{\text {in }} \rightarrow y_{1}\right) & \propto \exp \left[-\frac{1}{2}\left(y_{1}^{T}-y_{\text {in }}^{T} R_{0}^{T}\right) \Sigma_{1}^{-1}\left(y_{1}-R_{0} y_{\text {in }}\right)\right] \\
L_{\text {scat }}\left(y_{1} \rightarrow y_{2}=y_{\text {out }}\right) & \propto \exp \left[-\frac{1}{2}\left(y_{\text {out }}^{T}-y_{1}^{T} R_{1}^{T}\right) \Sigma_{2}^{-1}\left(y_{\text {out }}-R_{1} y_{1}\right)\right],
\end{aligned}
$$

with

$$
\Sigma_{1}=\left(\begin{array}{cc}
\sigma_{t_{1}}^{2} & \sigma_{t_{1} \theta_{1}}^{2} \\
\sigma_{t_{1} \theta_{1}}^{2} & \sigma_{\theta_{1}}^{2}
\end{array}\right), \Sigma_{2}=\left(\begin{array}{cc}
\sigma_{t_{2}}^{2} & \sigma_{t_{2} \theta_{2}}^{2} \\
\sigma_{t_{2} \theta_{2}}^{2} & \sigma_{\theta_{2}}^{2}
\end{array}\right), R_{0}=\left(\begin{array}{cc}
1 & u-u_{\text {in }} \\
0 & 1
\end{array}\right), R_{1}=\left(\begin{array}{cc}
1 & u_{\text {out }}-u \\
0 & 1
\end{array}\right) .
$$

The matrices $R_{0}$ and $R_{1}$ can be thought of as small angle rotation matrices and $\Sigma_{1}$ and $\Sigma_{2}$ are covariance matrices which quantify the amount of spatial and angular dispersion in a certain depth due to MCS. Their components can be calculated numerically. In this work, we used the integral expressions reported in Eqs. 7-9 and Eqs. 16-18 in (Schulte et al. 2008). They differ from those in (Williams 2004) only by the additional pre-factor. We report them here for completeness:

$$
\begin{aligned}
\sigma_{t_{1}}^{2} & =E_{0}^{2}\left(1+0.038 \ln \frac{u-u_{\text {in }}}{X_{0}}\right)^{2} \times \int_{u_{\text {in }}}^{u} \frac{\left(u-u_{\text {in }}\right)^{2}}{\beta^{2} p^{2}} \frac{\mathrm{d} u}{X_{0}} \\
\sigma_{\theta_{1}}^{2} & =E_{0}^{2}\left(1+0.038 \ln \frac{u-u_{\text {in }}}{X_{0}}\right)^{2} \times \int_{u_{\text {in }}}^{u} \frac{1}{\beta^{2} p^{2}} \frac{\mathrm{d} u}{X_{0}} \\
\sigma_{t_{1} \theta_{1}}^{2} & =E_{0}^{2}\left(1+0.038 \ln \frac{u-u_{\text {in }}}{X_{0}}\right)^{2} \times \int_{u_{\text {in }}}^{u} \frac{\left(u-u_{\text {in }}\right)}{\beta^{2} p^{2}} \frac{\mathrm{d} u}{X_{0}},
\end{aligned}
$$

with $E_{0}=13.6 \mathrm{MeV} / \mathrm{c}$ an empirical constant. The components of $\Sigma_{2}$ are obtained by replacing $u_{\text {in }}$ with $u$ and $u$ with $u_{\text {out }}$ in the above equations. The $1 / \beta^{2} p^{2}$ term depends on the protons energy and increases with depth. It is often approximated by a polynomial fit to MC simulated data in the context of proton CT reconstruction because Eqs. 9 . 11 can then be integrated analytically (Williams 2004). We will discuss this aspect in more detail in Sec. 2.8. The parameter $X_{0}$ is the material specific radiation length and its value is $36.1 \mathrm{~cm}$ for water. Smaller values, such as in bone tissue, lead to more scattering and thus larger entries in the covariance matrices $\Sigma_{1}$ and $\Sigma_{2}$. 
The MLP is obtained by maximizing Eq. 5 with respect to $y_{1}$ :

$$
\begin{aligned}
y_{\mathrm{MLP}}(u) & =\left(\Sigma_{1}^{-1}+R_{1}^{T} \Sigma_{2}^{-1} R_{1}\right)^{-1} \cdot\left(\Sigma_{1}^{-1} R_{0} y_{\text {in }}+R_{1}^{T} \Sigma_{2}^{-1} y_{\text {out }}\right) \\
& =R_{1}^{-1} \Sigma_{2}\left(R_{1}^{-1} \Sigma_{2}+\Sigma_{1} R_{1}^{T}\right)^{-1} \cdot R_{0} y_{\text {in }}+\Sigma_{1}\left(R_{1} \Sigma_{1}+\Sigma_{2}\left(R_{1}^{-1}\right)^{T}\right)^{-1} \cdot y_{\text {out }} .
\end{aligned}
$$

It is important to remember that the MLP is only an estimate of the proton path and the true trajectory will always differ from it. The uncertainty distribution around the MLP is (by construction) Gaussian and the covariance matrix is derived from the joint likelihood in Eq. 5 .

$$
\Sigma_{\mathrm{MLP}}(u)=\left(\Sigma_{1}^{-1}+R_{1}^{T} \Sigma_{2}^{-1} R_{1}\right)^{-1}=\Sigma_{1}\left(\Sigma_{2}\left(R_{1}^{-1}\right)^{T}+R_{1} \Sigma_{1}\right)^{-1} \Sigma_{2}\left(R_{1}^{-1}\right)^{T}
$$

The width of the uncertainty envelope (in the spatial domain) is given by the (1,1)-component of $\left(\Sigma_{\mathrm{MLP}}\right)_{1,1}$. The alternative forms on the right hand side of Eqs. 12 and 13 do not require inverting individual $\Sigma$-matrices which would otherwise diverge at the phantom entry or exit surface where one of them becomes zero.

\subsection{Set-up geometry}

In this section, we introduce relationships to account for the geometry of the imaging set-ups. We again refer to Fig. 2 for an overview sketch and a summary of the variables. All expressions tacitly make use of first order approximations of triangular functions, as do the existing MLP formalisms, because angular deflections due to MCS are small.

In a clinical implementation, the detector devices need to be placed at some minimum distance from the patient, for practical as well as security reasons. The authors of (Schulte et al. 2004) recommend a minimum distance of $10 \mathrm{~cm}$ between the trackers and the phantom/patient. From the practical point of view, more clearance might be needed to allow the detector to rotate around the patient and the couch. In comparison, the flat panel imagers in several commercial cone beam CT scanners have a distance of $40-50 \mathrm{~cm}$ to the centre of rotation, which implies a distance of 30-40 cm between the detector and the patient head surface (which we denote with $d_{\text {entry }}$ and $\left.d_{\text {exit }}\right)$. In this work, we explore the impact of these geometrical parameters in the range between $2 \mathrm{~cm}$ and $40 \mathrm{~cm}$.

We assume the protons to propagate on straight lines in the air surrounding the patient because the scattering probability is very low (radiation length of air is about $3 \times 10^{4} \mathrm{~cm}$ ). We denote with $y_{\text {in,d }}$ the parameter vector measured in the tracker plane which translates into a vector $y_{\text {in }}$ at the entrance surface of the phantom as

$$
y_{\text {in }}=S_{\text {in }} \cdot y_{\text {in,d }} \quad \text { with } \quad S_{\text {in }}=\left(\begin{array}{cc}
1 & d_{\text {entry }} \\
0 & 1
\end{array}\right) .
$$

Similarly, for the exit vector we write

$$
y_{\text {out }, \mathrm{d}}=S_{\text {out }} \cdot y_{\text {out }} \quad \text { with } \quad S_{\text {out }}=\left(\begin{array}{cc}
1 & d_{\text {exit }} \\
0 & 1
\end{array}\right),
$$

where $y_{\text {out,d }}$ is the exit coordinate vector in the tracker plane and $y_{\text {out }}$ is its back projection onto the phantom surface.

\subsection{Extended formalism}

The expression in Eq. 13 for the width of the uncertainty envelope assumes $y_{\text {in }}$ and $y_{\text {out }}$ to be precisely known. In this section, we extend the formalism to account for experimental uncertainties in $y_{\text {in }}$ and $y_{\text {out }}$.

From the statistical point of view, the entry and exit parameters measured by the tracker devices of a single tracking set-up provide an estimate of their true values with some degree of 
uncertainty. In the following, we add the tilde symbol when referring to measured estimates and leave it away to indicate their true values. We model the uncertainty with a Gaussian distribution because it often describes well the statistical errors in experimental practice and it simplifies the mathematical equations. The likelihood of the measured values $\tilde{y}_{\text {in,d }}$ and $\tilde{y}_{\text {out,d }}$ to be true is

$$
\begin{aligned}
L_{\text {meas }}\left(\tilde{y}_{\text {in }, \mathrm{d}}, y_{\text {in }, \mathrm{d}}\right) & \propto \exp \left[-\frac{1}{2}\left(\tilde{y}_{\text {in }, \mathrm{d}}-y_{\text {in }, \mathrm{d}}\right)^{T} \Sigma_{\text {in }}^{-1}\left(\tilde{y}_{\text {in }, \mathrm{d}}-y_{\text {in }, \mathrm{d}}\right)\right] \\
\Rightarrow L_{\text {meas }}\left(\tilde{y}_{\text {in }}, y_{\text {in }}\right) & \propto \exp \left[-\frac{1}{2}\left(\tilde{y}_{\text {in }}-y_{\text {in }}\right)^{T}\left(S_{\text {in }} \Sigma_{\text {in }} S_{\text {in }}^{T}\right)^{-1}\left(\tilde{y}_{\text {in }}-y_{\text {in }}\right)\right] \\
L_{\text {meas }}\left(\tilde{y}_{\text {out }, \mathrm{d}}, y_{\text {out }, \mathrm{d}}\right) & \propto \exp \left[-\frac{1}{2}\left(\tilde{y}_{\text {out }, \mathrm{d}}-y_{\text {out }, \mathrm{d}}\right)^{T} \Sigma_{\text {out }}^{-1}\left(\tilde{y}_{\text {out }, \mathrm{d}}-y_{\text {out }, \mathrm{d}}\right)\right] \\
\Rightarrow L_{\text {meas }}\left(\tilde{y}_{\text {out }}, y_{\text {out }}\right) & \propto \exp \left[-\frac{1}{2}\left(\tilde{y}_{\text {out }}-y_{\text {out }}\right)^{T} S_{\text {out }}^{T} \Sigma_{\text {out }}^{-1} S_{\text {out }}\left(\tilde{y}_{\text {out }}-y_{\text {out }}\right)\right],
\end{aligned}
$$

where $\Sigma_{\text {in }}$ and $\Sigma_{\text {out }}$ are the covariance matrices which describe the experimental uncertainty. The above expressions can be used to extend Eq. 5 by marginalising over all possible true entry and exit parameters weighted by their likelihood:

$$
\begin{aligned}
& L\left(y_{1}, y_{2}=\tilde{y}_{\text {out }} \mid \tilde{y}_{\text {in }}\right)= \\
& \quad=\iint L_{\text {meas }}\left(\tilde{y}_{\text {in }}, y_{\text {in }}\right) L_{\text {scat }}\left(y_{\text {in }} \rightarrow y_{1}\right) L_{\text {scat }}\left(y_{1} \rightarrow y_{2}=y_{\text {out }}\right) L_{\text {meas }}\left(\tilde{y}_{\text {out }}, y_{\text {out }}\right) \mathrm{d} y_{\text {in }} \mathrm{d} y_{\text {out }} \\
& \quad=\int L_{\text {meas }}\left(\tilde{y}_{\text {in }}, y_{\text {in }}\right) L_{\text {scat }}\left(y_{\text {in }} \rightarrow y_{1}\right) \mathrm{d} y_{\text {in }} \times \int L_{\text {scat }}\left(y_{1} \rightarrow y_{2}=y_{\text {out }}\right) L_{\text {meas }}\left(\tilde{y}_{\text {out }}, y_{\text {out }}\right) \mathrm{d} y_{\text {out }} .
\end{aligned}
$$

This is the likelihood that a proton has passed at $y_{1}$ in some depth $u$ given the measurements $\tilde{y}_{\text {in }}$ and $\tilde{y}_{\text {out }}$. The two integrals in Eq. 18 can be solved analytically because all terms are Gaussian likelihood functions. One obtains:

$$
L\left(y_{1}, y_{2}=\tilde{y}_{\text {out }} \mid \tilde{y}_{\text {in }}\right) \propto \exp \left[-\frac{1}{2}\left(y_{1}-y_{\mathrm{MLP}}\right)^{T} \Sigma_{\mathrm{MLP}}^{-1}\left(y_{1}-y_{\mathrm{MLP}}\right)\right],
$$

where

$$
\begin{aligned}
\Sigma_{\mathrm{MLP}}(u) & =C_{1}\left(C_{1}+C_{2}\right)^{-1} C_{2} \\
y_{\mathrm{MLP}}(u) & =C_{2}\left(C_{1}+C_{2}\right)^{-1} R_{0} S_{\mathrm{in}} \cdot \tilde{y}_{\mathrm{in}, \mathrm{d}} \\
& +C_{1}\left(C_{1}+C_{2}\right)^{-1} R_{1}^{-1} S_{\text {out }}^{-1} \cdot \tilde{y}_{\text {out }, \mathrm{d}}
\end{aligned}
$$

with

$$
\begin{aligned}
& C_{1}=R_{0} S_{\text {in }} \Sigma_{\text {in }} S_{\text {in }}^{T} R_{0}^{T}+\Sigma_{1} \\
& C_{2}=R_{1}^{-1} S_{\text {out }}^{-1} \Sigma_{\text {out }}\left(S_{\text {out }}^{-1}\right)^{T}\left(R_{1}^{-1}\right)^{T}+R_{1}^{-1} \Sigma_{2}\left(R_{1}^{-1}\right)^{T}
\end{aligned}
$$

The width $\sigma_{\text {MLP }}$ of the uncertainty envelope around the MLP in the spatial domain is given by the $(1,1)$ component of the uncertainty matrix:

$$
\sigma_{\mathrm{MLP}}(u)=\left(\Sigma_{\mathrm{MLP}}(u)\right)_{1,1} .
$$

We remark that both, $\Sigma_{\text {MLP }}$ and $y_{\mathrm{MLP}}$, depend on depth, but only $y_{\mathrm{MLP}}$ depends on the entrance and exit coordinates. At the same time, $y_{\mathrm{MLP}}$ also depends on the parametrisation of the detector uncertainties through the $\Sigma_{\text {in }}$ and $\Sigma_{\text {out }}$ matrices. We note that under the assumption of perfect trackers, $\Sigma_{\text {in }} \rightarrow 0$ and $\Sigma_{\text {out }} \rightarrow 0$, and Eqs. 20 and 21 become the "conventional" expressions (Eqs. 12 and 13 ). 


\subsection{Detector uncertainties in single tracking set-ups}

Single tracking set-ups determine a proton's propagation angle by measuring its position in two consecutive tracking detectors with a known distance from each other. One pair of trackers is needed for each of the two directions transversal to the main beam axis. The precision of such a measurement depends on the resolution of the strips (or fibres) as well as on the distance between the two trackers. Scattering in the tracker proximal to the phantom leads to additional angular uncertainty depending on the amount and kind of material (the so-called material budget $\left.x / X_{0}\right)$. The impact of these parameters on the MLP precision in single tracking set-ups has been investigated elsewhere (Bopp et al. 2014, Penfold et al. 2011) although the authors do not provide an analytical expression to calculate the uncertainty of the entry/exit position and angle.

We denote with $p=\left(p_{1}, p_{2}\right)$ the two transversal positions measured by one pair of tracking detectors, with $d_{\mathrm{T}}$ the distance between the two trackers, and with $\sigma_{\mathrm{p}}$ their resolution. On the upstream side of the phantom, the proton coordinates $y_{\mathrm{in}, \mathrm{d}}=\left(t_{\mathrm{in}, \mathrm{d}}, \theta_{\mathrm{in}, \mathrm{d}}\right)$ are obtained as

$$
y_{\text {in }, \mathrm{d}}=T_{\mathrm{in}} \cdot p \quad \text { with } \quad T_{\mathrm{in}}=\left(\begin{array}{cc}
0 & 1 \\
-1 / d_{\mathrm{T}} & 1 / d_{\mathrm{T}}
\end{array}\right),
$$

and on the downstream side as

$$
y_{\text {out }, \mathrm{d}}=T_{\text {out }} \cdot p \quad \text { with } \quad T_{\text {out }}=\left(\begin{array}{cc}
1 & 0 \\
-1 / d_{\mathrm{T}} & 1 / d_{\mathrm{T}}
\end{array}\right) .
$$

We assume the tracker resolution can be adequately described with two independent Gaussian uncertainty distributions and a corresponding diagonal covariance matrix $\Sigma_{p}=\sigma_{p}^{2} \cdot \operatorname{diag}(1,1)$ (Bopp et al. 2014). We describe the effect of scattering on the tracker facing the phantom as additional covariance matrix using Eq. 12 from (Lynch \& Dahl 1991):

$$
\Sigma_{\mathrm{sc}}=\left(\begin{array}{cc}
0 & 0 \\
0 & \sigma_{\mathrm{sc}}^{2}
\end{array}\right) \quad \text { with } \quad \sigma_{\mathrm{sc}}=\frac{13.6 \mathrm{MeV}}{\beta(E) p(E)} \sqrt{\frac{x}{X_{0}}}\left[1+0.038 \ln \left(\frac{x}{X_{0}}\right)\right] .
$$

The covariance matrices with respect to $y_{\text {in,d }}$ and $y_{\text {out,d }}$, respectively, are

$$
\Sigma_{\text {in }}=\sigma_{p}^{2} T_{\text {in }} \cdot T_{\text {in }}^{T}+\Sigma_{\text {sc }} \quad \text { and } \quad \Sigma_{\text {out }}=\sigma_{p}^{2} T_{\text {out }} \cdot T_{\text {out }}^{T}+\Sigma_{\text {sc }} .
$$

Note that the matrix in Eq. 27 by itself would not be invertible, but the full expressions in Eq. 28 are. As characteristic values representative of a single tracking set-up, we used $\sigma_{p}=$ $0.15 \mathrm{~mm}, x / X_{0}=5 \times 10^{-3}$, and $d_{\mathrm{T}}=10 \mathrm{~cm}$ (Amaldi et al. 2011, Scaringella et al. 2014, Penfold et al. 2011, Coutrakon et al. 2014).

\subsection{Application of the formalism to integral mode set-ups}

The MLP formalism has originally been developed in the context of single tracking set-ups and, for the sake of comprehensibility, we have remained within this frame so far when extending the equations to account for the set-up geometry and for experimental uncertainties. In this section, we explain how the equations can be reinterpreted in a meaningful way to provide a figure of merit of the spatial resolution achievable with integral mode proton imaging set-ups.

In single proton tracking, the MLP path is estimated based on the entrance and exit parameters measured by the trackers. The standard deviation of the uncertainty envelope quantifies by how much the position $t_{1}$ in a certain depth $u$ estimated by the MLP deviates from the true proton trajectory on average over many registered events used in the tomographic reconstruction. In this sense it quantifies the accuracy of the projection model, i.e., of the MLP.

In integral mode set-ups, an ensemble of protons traverses the phantom and is captured in the detector. Therefore, the averaging is of physical nature and essentially takes place already 
during image acquisition. In a PBS + range telescope set-up, the MLP is simply a straight line (as long as tissue heterogeneities are neglected) and the beam width quantifies how far away from the centre of the beam the protons which contribute to the measured signal can have passed. From a statistical point of view, the beam width, i.e., the size of the uncertainty envelope, quantifies the accuracy of the projection model (straight line MLP) compared to the true finite size beam.

In a PBS + pixel detector set-up, there are $N \times M$ projection lines (MLPs) per projection angle, where $N$ is the number of pencil beam spots and $M$ the number of detector pixels. The effective number of projection lines which can be used in a tomographic reconstruction algorithm will certainly be lower because pixels too far away from the beam centre do not capture enough protons to produce a sufficient signal.

From the imaging point of view, the $\sigma_{\mathrm{MLP}}$ parameter in any case gives a characteristic measure of the spatial resolution in the reconstructed image: the larger its value the blurrier the image. Following this argumentation, Eq. 20 becomes a polyvalent description of all four proton imaging set-ups considered in this work by suitably adjusting the components of $\Sigma_{\text {in }}$ and $\Sigma_{\text {out }}$ to reflect the beam's phase space and the detector properties, respectively. The tracker planes indicated in Fig. 2 have a different meaning for the integrated mode set-ups: the entrance tracker plane can be viewed as the isocentre plane (where the nominal beam parameters typically refer to) and the exit tracker plane corresponds to the detector surface. Consequently, $d_{\text {entry }}$ specifies the distance of the phantom entrance surface from isocentre and $d_{\text {exit }}$ the detector distance from the phantom exit surface.

In PBS, the proton beam is steered by deflection magnets typically a few meters upstream from the nozzle and the uncertainty associated with the entrance position of the protons is given by the beam spot size. In double scattering, protons traverse two subsequent scattering surfaces at some distance upstream from the isocentre which spread the proton beam into a cone beam shaped field whose edges are cut away by collimators (Grusell et al. 1994, Paganetti 2012a). Their position when entering the phantom is essentially unknown. In both cases, scattering due to components along the beam line (e.g., second scattering surface, exit windows, monitoring units etc.) leads to a certain degree of angular confusion. An exact mathematical model of the beam geometry can be complex and is out of scope for this work.

We used a virtual point source model to describe the beam geometry and to take into account the beam divergence. In this framework, $\Sigma_{\text {in }}$ becomes

$$
\Sigma_{\mathrm{in}}=\left(\begin{array}{cc}
1 & 0 \\
1 / d_{\mathrm{s}} & 1
\end{array}\right) \cdot\left(\begin{array}{cc}
\sigma_{t_{\mathrm{in}}}^{2} & 0 \\
0 & 0
\end{array}\right) \cdot\left(\begin{array}{cc}
1 & 1 / d_{\mathrm{s}} \\
0 & 1
\end{array}\right)+\left(\begin{array}{cc}
0 & 0 \\
0 & \sigma_{\theta_{\mathrm{in}}}^{2}
\end{array}\right) .
$$

The uncertainty parameter $\sigma_{t_{\mathrm{in}}}$ represents the (Gaussian) beam size in the isocentre plane and $\sigma_{\theta_{\text {in }}}$ quantifies the angular confusion. The parameter $d_{\mathrm{s}}$ describes the upstream distance of the point source from the isocentre plane. We set $d_{\mathrm{s}}=200 \mathrm{~cm}$ as figure of merit having in mind common values of the source axis distance in proton beam lines. Note that a parallel beam geometry can easily be recovered by letting $d_{\mathrm{s}} \rightarrow \infty$, i.e., setting $\Sigma_{\mathrm{in}}=\operatorname{diag}\left(\sigma_{t_{\mathrm{in}}}^{2}, \sigma_{\theta_{\mathrm{in}}}^{2}\right)$.

On the exit side, the PBS + range telescope set-up provides no information at all on proton position and angle. We model this numerically by setting both, $\sigma_{\text {tout }_{\text {out }}}$ and $\sigma_{\theta_{\text {out }}}$, to very large numbers $\left(20 \mathrm{~cm}\right.$ and $\left.45^{\circ}\right)$. In the two set-ups employing a pixel detector, the impact angle of the protons on the detector surface is unknown, so that again we set $\sigma_{\theta_{\text {out }}}$ to a large number $\left(45^{\circ}\right)$. The impact position is known from the pixel coordinate and we set $\sigma_{\mathrm{t}_{\mathrm{out}}}$ to $0.5 \mathrm{~mm}$ as a figure of merit. The exact value depends on factors such as the type of flat panel detector and the degree of interplay between adjacent pixels.

\subsection{Figure of merit for comparison of the spatial resolution}

The width of the uncertainty envelope $\sigma_{\mathrm{MLP}}(u)$ depends on depth so that objects will be more or less blurred in a proton radiography depending on their location within the phantom. In 
single tracking set-ups, for example, the envelope is generally wider in the central part of the phantom.

As a figure of merit for the spatial resolution, we used the spatial frequency at which the modular transfer function (MTF) of a density step located at some depth $u$ within the phantom decays below 10\% (Richard et al. 2012). Similar methods have been employed by other authors in the context of proton imaging (Hansen et al. 2016, Hansen et al. 2014, Seco et al. 2013, Plautz et al. 2016).

Within the Gaussian approximation of MCS, the edge spread function (ESF) of a thin dense slab at depth $u$ is an error function,

$$
\operatorname{ESF}(t ; u) \propto \frac{1}{2}\left(1+\operatorname{erf}\left(\frac{t}{\sqrt{2} \sigma_{\mathrm{MLP}}(u)}\right)\right)
$$

where $t$ denotes the transversal coordinate (see Fig. 2). We will use this to graphically illustrate the effect of spatial blurring. Its derivative yields the line spread function (LSF) and the modulus of its Fourier transform (again a Gaussian) is the depth dependent MTF. The spatial frequency at which the MTF decays below $10 \%$ of its maximum value is

$$
f_{10 \%}(u)=\frac{\sqrt{2 \ln 10}}{2 \pi} \frac{1}{\sigma_{\mathrm{MLP}}(u)}, \quad \text { with } \quad \frac{\left|M T F\left(f_{10 \%}\right)\right|}{|M T F(0)|}=\frac{1}{10} .
$$

In the results, we report the average value of $f_{10 \%}(u)$ along the phantom depth $f_{10 \%}^{\text {ave }}$ as well as its minimum $f_{10 \%}^{\min }$ and maximum $f_{10 \%}^{\max }$ values. We remark that $f_{10 \%}$ serves as characteristic figure of merit of each imaging set-up while it does not explicitly quantify the spatial resolution of a reconstructed tomographic image, as this depends, e.g., on the kind of reconstruction algorithm.

\subsection{Numerical treatment of proton energy loss}

The $1 / \beta^{2} p^{2}$ term in Eqs. 9 through 11 takes into account the energy dependence of the proton scattering within the phantom. It must be appropriately parametrised numerically to perform the integration.

In single tracking set-ups, protons range out beyond the trackers in the energy detector behind them. In principle, any initial beam energy can be used provided that the protons traverse the patient and reach the detector. The same holds true for a PBS+range telescope set-up. Note, however, that the choice of the initial energy will have an impact on the density resolution in the proton CT image in single tracking (Schulte et al. 2005). For imaging set-ups which make use of a single plane pixel detector the situation is different because the WET measurement principle poses a constraint on the initial proton energy: It has to be selected such that the proton range (in water) is equivalent to the phantom WET plus an additional absorber slab between the phantom and the detector. The latter is necessary to guarantee that the patient is not (accidentally) exposed to high dose in case the protons range out earlier than predicted when generating the irradiation plan. We assumed a slab thickness of $2 \mathrm{~cm}$ in our analysis which seems a reasonable minimal requirement. The final results in terms of $\bar{\sigma}_{\mathrm{MLP}}$ do not appreciably $(\leq 10 \%)$ change for larger values up to several $\mathrm{cm}$.

This constraint between energy and WET prompted us to use the analytical expression proposed by (Bortfeld \& Schlegel 1996) to model the kinetic energy $E$ (in MeV) as a function of depth (in $\mathrm{cm})$,

$$
E(u)=\left(\frac{R-u}{\alpha}\right)^{1 / p},
$$

where $R=\mathrm{WET}_{\text {phantom }}+2 \mathrm{~cm}$ is the range of protons stopping in the flat panel detector. Following Eq. 28 of (Schulte et al. 2008), we then calculated $1 / \beta^{2} p^{2}$ as

$$
\frac{1}{\beta^{2} p^{2}}(u)=\frac{\left(E(u)+E_{p}\right)^{2} c^{2}}{\left(E(u)+2 E_{p}\right)^{2} E^{2}(u)},
$$


and integrated Eqs. 9 through 11 numerically. In the MLP formalisms used for single tracking set-ups and the related tomographic reconstruction, the $1 / \beta^{2} p^{2}$ term is approximated by a polynomial (of 5 th order), as initially proposed by (Williams 2004). Such a parametrisation stems from the fact that the integrals in Eqs. 9 through 11 can then be solved analytically. The use of polynomials saves computation time when reconstructing the $3 \mathrm{D}$ image. On the other hand, computational performance was no concern for our study and Eq. 32 is very intuitive for our purpose because it explicitly contains the parameter $\mathrm{WET}_{\text {phantom }}$.

We performed a simple Geant4 v10.3.2 MC simulation (Agostinelli et al. 2003) via Gate (Jan et al. 2011, Sarrut et al. 2014) to determine the best choice of the parameters $\alpha$ and $p$ in Eq. 32. We had a mono-energetic proton beam with $\mathrm{FWHM}=8 \mathrm{~mm}$ impinge onto a large block of water and scored the proton energy as a function of depth in $1 \mathrm{~mm}$ steps. We determined the range $R$ from the integrated depth dose profiles using the $80 \%$ distal fall-off criterion. By fitting $E_{\text {beam }}=(R / \alpha)^{1 / p}$ to the simulated data for different beam energies between $50 \mathrm{MeV}$ and $200 \mathrm{MeV}$, we established $p=1.5$ and $\alpha=0.01$.

Many flat panel detectors contain a conversion layer made of scintillator material in which incoming X-rays and in our case protons generate light which is then captured by a detector array below. Others make use of direct conversion into electric charge without a scintillator layer. In any case, protons will experience some degree of scattering in the flat panel and the additional (safety) absorber slab in front of it. Because it is not straightforward to model these effects, especially without assuming specifics about the detector, we neglect this additional scattering and take into account only the energy loss in the absorber slab by setting $R=$ $\mathrm{WET}_{\text {phantom }}+2 \mathrm{~cm}$. As a result, the effective position uncertainty of a true set-up might be larger than the nominal pixel size. In this sense, our figures of merit of $\bar{\sigma}_{\mathrm{MLP}}$ for the PBS+pixel detector and passive field+pixel detector set-ups should be considered as lower limit values.

\section{$2.9 \quad$ MC simulations}

In order to verify the analytical calculations presented in the previous sections, we performed MC simulations using Geant4/Gate. The phantom used in all simulations was a $15 \mathrm{~cm}$ thick block of water with dimensions $50 \times 50 \mathrm{~cm}^{2}$ in the transversal plane. We positioned one phase space actor in front of and one behind the phantom which recorded position and angle of the protons. As a moderate choice of set-up parameters, we selected $d_{\text {entry }}=d_{\text {exit }}=15 \mathrm{~cm}$. The maximum step length was set to $2 \mathrm{~mm}$ inside the phantom to guarantee a fine sampling of the proton trajectories. Two different physics lists were used to model the physical processes: emstandard to keep only electromagnetic interactions and $Q G S P$-BIC to include also nuclear interactions.

Table 1 summarises the simulation parameters for each set-up. Protons were sent in a $20 \times 20 \mathrm{~cm}^{2}$ square field to model double scattering and as a Gaussian beam with $8 \mathrm{~mm}$ FWHM spot size for PBS. In all simulations, the proton beam/field was diverging with the focus point $200 \mathrm{~cm}$ upstream from the entrance surface of the phantom. The single tracking set-up did not need a specific simulation as data from other set-ups could be used. We set the initial energy to $200 \mathrm{MeV}$ for the range telescope set-up and chose the beam energy such that $R=$ $\mathrm{WET}_{\text {phantom }}+2 \mathrm{~cm}$ (see Sec. 2.8) for set-ups involving a pixel detector. In post-processing, protons were selected according to their position recorded $15 \mathrm{~cm}$ behind the phantom to mimic the pixel geometry. Therefore, certain set-ups required more protons because over $99 \%$ of them would be filtered out.

\section{RESULTS}

In the following, we present the results obtained through our amended analytical formalism (Sec.2.4) as well as from MC simulations (Sec. 2.9). Specifically, we show uncertainty envelopes 
Table 1: Geant4 simulation parameters for each set-up.

\begin{tabular}{c|c|c|c} 
Set-up & Source geometry & Initial energy & Number of protons \\
\hline \hline Passive field + pixel detector & $20 \times 20 \mathrm{~cm}^{2}$ square field & $157 \mathrm{MeV}$ & $10^{6}$ \\
\hline PBS + range telescope & Gaussian beam $\sigma_{\tau}=3.4 \mathrm{~mm}$ & $200 \mathrm{MeV}$ & $10^{5}$ \\
\hline PBS + pixel detector & Gaussian beam $\sigma_{\tau}=3.4 \mathrm{~mm}$ & $157 \mathrm{MeV}$ & $10^{6}$ \\
\hline
\end{tabular}

Table 2: Uncertainty parameters used to characterise the proton imaging set-ups.

\begin{tabular}{l||c|c|c|c} 
set-up \parameter & $\sigma_{t_{\text {in }}}$ & $\sigma_{\theta_{\text {in }}}$ & $\sigma_{t_{\text {out }}}$ & $\sigma_{\theta_{\text {out }}}$ \\
\hline \hline Single tracking idealised & 0 & 0 & 0 & 0 \\
\hline PBS + range telescope idealised & 0 & 0 & $\rightarrow \infty$ & $\rightarrow \infty$ \\
\hline PBS + pixel detector idealised & 0 & 0 & 0 & $\rightarrow \infty$ \\
\hline passive scattering + pixel detector idealised & $\rightarrow \infty$ & 0 & 0 & $\rightarrow \infty$ \\
\hline \hline Single tracking (see Sec. 28 & $0.15 \mathrm{~mm}$ & $3 \mathrm{mrad}$ & $0.15 \mathrm{~mm}$ & $3 \mathrm{mrad}$ \\
\hline Single tracking without angle measurement & $0.5 \mathrm{~mm}$ & $15 \mathrm{mrad}$ & $0.5 \mathrm{~mm}$ & $\rightarrow \infty\left(45^{\circ}\right)$ \\
\hline PBS + range telescope & $8 / 2.35 \mathrm{~mm}$ & $0.1 \mathrm{mrad}$ & $20 \mathrm{~cm}$ & $\rightarrow \infty\left(45^{\circ}\right)$ \\
\hline passive scattering + pixel detector & $20 \mathrm{~cm}$ & $15 \mathrm{mrad}$ & $0.5 \mathrm{~mm}$ & $\rightarrow \infty\left(45^{\circ}\right)$ \\
\hline PBS + pixel detector & $8 / 2.35 \mathrm{~mm}$ & $0.1 \mathrm{mrad}$ & $0.5 \mathrm{~mm}$ & $\rightarrow \infty\left(45^{\circ}\right)$
\end{tabular}

for the four set-up types as well as the figure of merit of the spatial resolution (Sec. 2.7) for different geometrical configurations and detector uncertainties. For the single tracking and the range telescope set-ups, we used a range corresponding to $200 \mathrm{MeV}$ protons in water to model the energy dependent term in the MCS formulæ (see Sec. 2.8). For the other two, the range was always set to $R=\mathrm{WET}_{\text {phantom }}+2 \mathrm{~cm}$. To generate the uncertainty envelope outside of the phantom, we propagated the covariance matrix $\Sigma_{\text {MLP }}$ backward from the entrance surface and forward from the exit surface, neglecting scattering in air. The size of the uncertainty envelope, $\sigma_{\mathrm{MLP}}(u)$, does not depend on the shape of the MLP, as pointed out in Sec. 2.4. For the sake of simpler illustration, we therefore depict a straight MLP along the isocentric beam axis.

\subsection{Comparison with MC simulations}

We simulated proton trajectories in water as explained in Sec. 2.9 to verify the conclusions based on our analytical calculations. The results are shown in the upper row of Fig. 3 where the yellow envelopes were obtained simulating MCS only and the blue ones with "all" interactions including elastic and inelastic nuclear scattering enabled. The dashed envelope shows the analytically calculated $\sigma_{\mathrm{MLP}}(u)(\mathrm{Eq} \cdot 24)$. We set $\mathrm{WET}_{\text {phantom }}=15 \mathrm{~cm}$ and $d_{\text {entry }}=d_{\text {exit }}=15 \mathrm{~cm}$ as in the MC simulation. For the $\Sigma_{\text {in }}$ and $\Sigma_{\text {out }}$ matrices, we used the parameters reported in the lower half of Tab. 2 .

The lower row of Fig. 3 depicts the relative difference between $\sigma_{\mathrm{MLP}}(u)$ obtained from the MC simulations and calculated through our amended analytical formalism. From the MC simulations, $\sigma_{\mathrm{MLP}}(u)$ was estimated as standard deviation of the simulated proton trajectories. The coloured blue halo gives the standard error of the standard deviation of the simulated proton trajectories.

\subsection{Idealised set-up parameters}

We used a set of idealised set-up parameters for each of the set-ups. Their purpose was to provide a reference case in which MCS was the only source of uncertainty while neglecting set-up related factors. Specifically, where appropriate, we assumed perfectly precise trackers, an infinitely thin pencil beam, infinitely small pixels, and a proton field which follows a perfect 

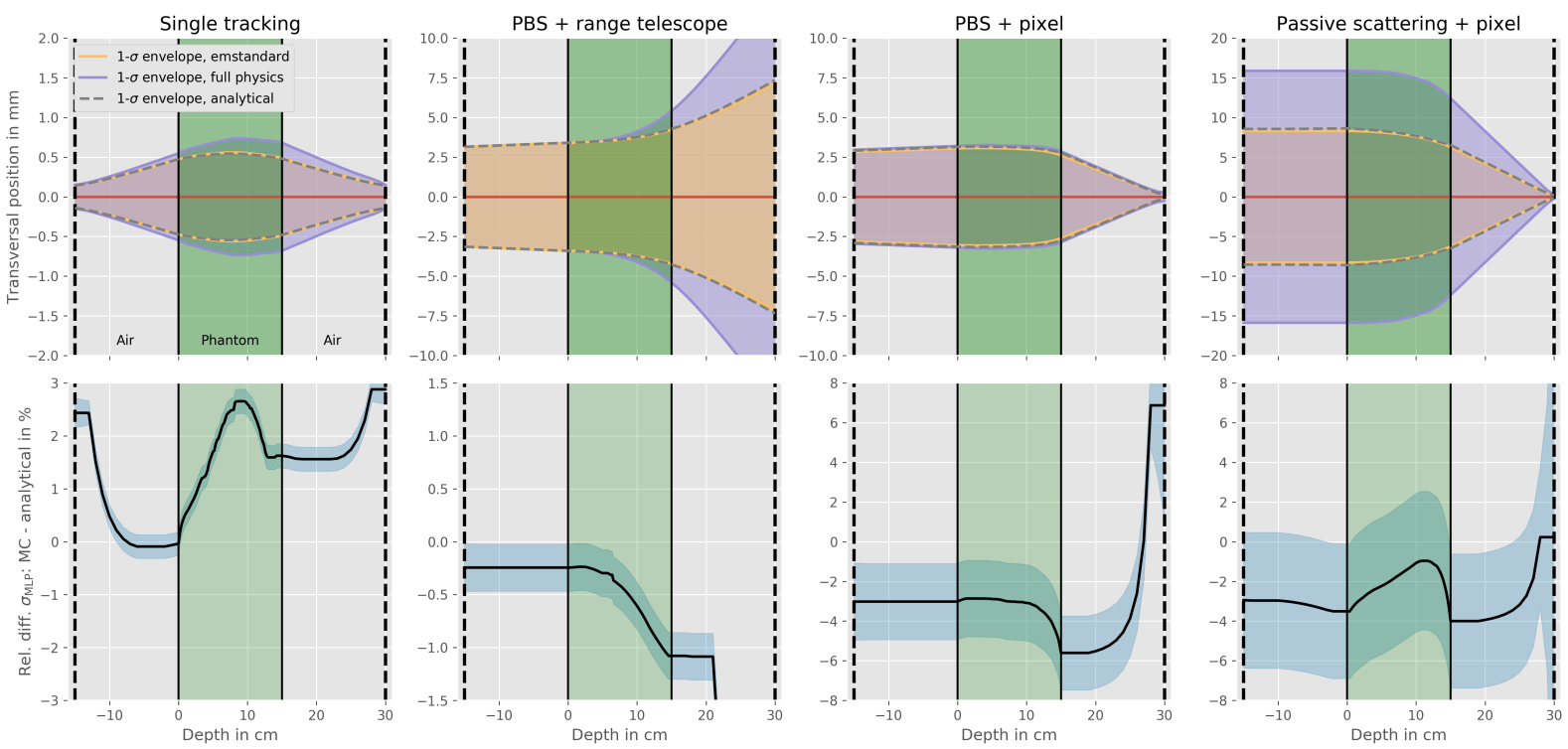

Figure 3: Upper row: Uncertainty envelopes obtained with Geant4/Gate simulation with the emstandard physics list (yellow) and the QGSP-BIC full physics list (blue). The dashed envelope shows the analytically calculated $\sigma_{\mathrm{MLP}}(u)$ (Eq. 24). Note the different scales used for the y-axis. Lower row: Relative difference between the analytically calculated and the MC simulated values of $\sigma_{\mathrm{MLP}}(u)$. The blue halo gives the statistical uncertainty due to the limited number of selected events in the MC simulation.

cone beam geometry without angular confusion (i.e, no residual scattering due to elements along the beam line). The parameters are summarised in the upper half of Table 2 . Figure 4 presents an overview of the uncertainty envelopes for the four set-ups under idealised conditions. We set $\mathrm{WET}_{\text {phantom }}=15 \mathrm{~cm}$ and $d_{\text {entry }}=d_{\text {exit }}=15 \mathrm{~cm}$ as moderate choice of geometric parameters.

\subsection{Non-idealised geometric set-up parameters}

Fig. 5 illustrates examples of uncertainty envelopes under non-idealised conditions for different distances $d_{\text {entry }}$ and $d_{\text {exit }}$ and phantom thickness values $\mathrm{WET}_{\text {phantom }}$, and Fig. 6 shows the dependence of the spatial resolution $f_{10 \%}$ on the distance between detector/trackers and phantom (left) and on the phantom thickness (right). For the $\Sigma_{\text {in }}$ and $\Sigma_{\text {out }}$ matrices, we used the parameters reported in the lower half of Tab. 2. The value $\sigma_{\theta_{\text {in }}}=15 \mathrm{mrad}$ corresponds to the angular confusion created by a $1 \mathrm{~mm}$ lead foil in double scattering.

For completeness, we have calculated the spatial resolution for single tracking set-ups which do not measure the protons' propagation angle (violet curve in Fig. 6). We set $\Sigma_{\text {in }}=\operatorname{diag}\left(\sigma_{t_{\text {in }}}, \sigma_{\theta_{\text {in }}}\right)$ and $\Sigma_{\text {out }}=\operatorname{diag}\left(\sigma_{t_{\text {out }}}, \sigma_{\theta_{\text {out }}}\right)$, with $\sigma_{t_{\text {in }}}=\sigma_{t_{\text {out }}}=0.5 \mathrm{~mm}$ representative of the width of scintillating fibres, $\sigma_{\theta_{\text {in }}}=15 \mathrm{mrad}$ a figure of merit for the angular confusion, and $\sigma_{\theta_{\text {out }}} \rightarrow \infty$ because no angular information is measured.

Figure 7 intends to provide a more intuitive understanding of the degree of blurring observable in the four set-ups. The plots show the (back-projected) profile which would be observed with a density step (e.g., half a slab of bone-like material) transversal to the beam axis inserted at some depth within the phantom. Within the Gaussian approximation of MCS, such a profile is described by an error function (see Sec. 2.7).

\subsection{Pencil beam spot size and pixel size}

In the left panel of Fig. 8, we have calculated the spatial resolution $f_{10 \%}$ as a function of beam spot size for the two PBS-based set-ups. The phantom thickness was $15 \mathrm{~cm}$. The right panel of 

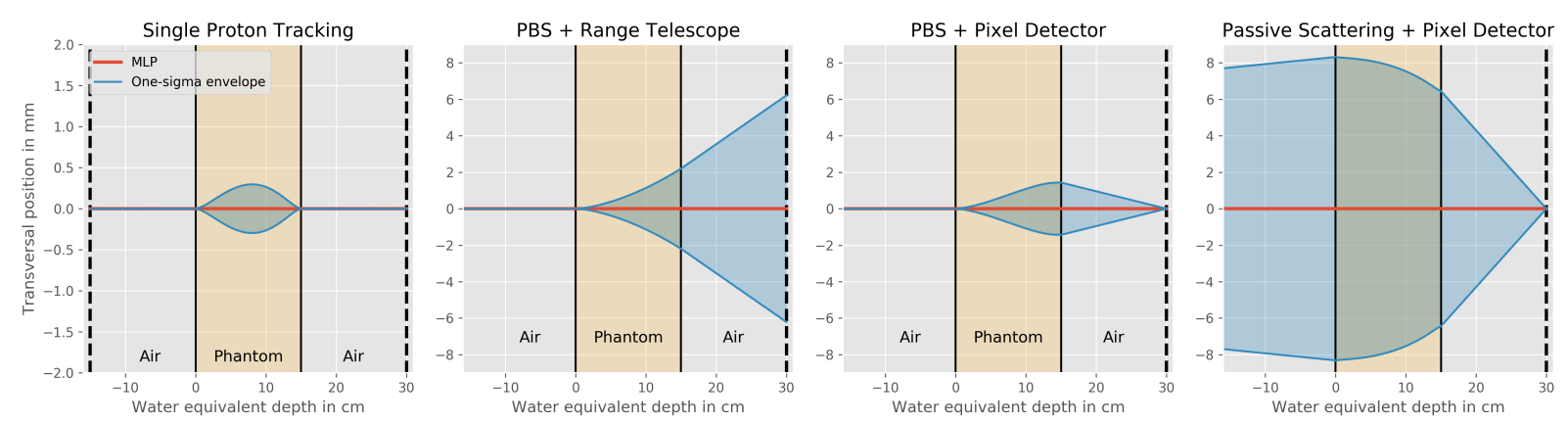

Figure 4: Uncertainty envelopes of idealised set-ups calculated using Eq. 20. The protons propagate from left to right and the origin of the depth coordinate refers to the entrance surface of the phantom. For the single tracking set-up, the two vertical dashed lines indicate the proton tracker pairs. For the other three set-ups, the vertical dashed line to the right of the phantom refers to the detector, although this is for reference purpose only in the case of the range telescope which does not provide spatial information. Note the different scale in the leftmost panel.
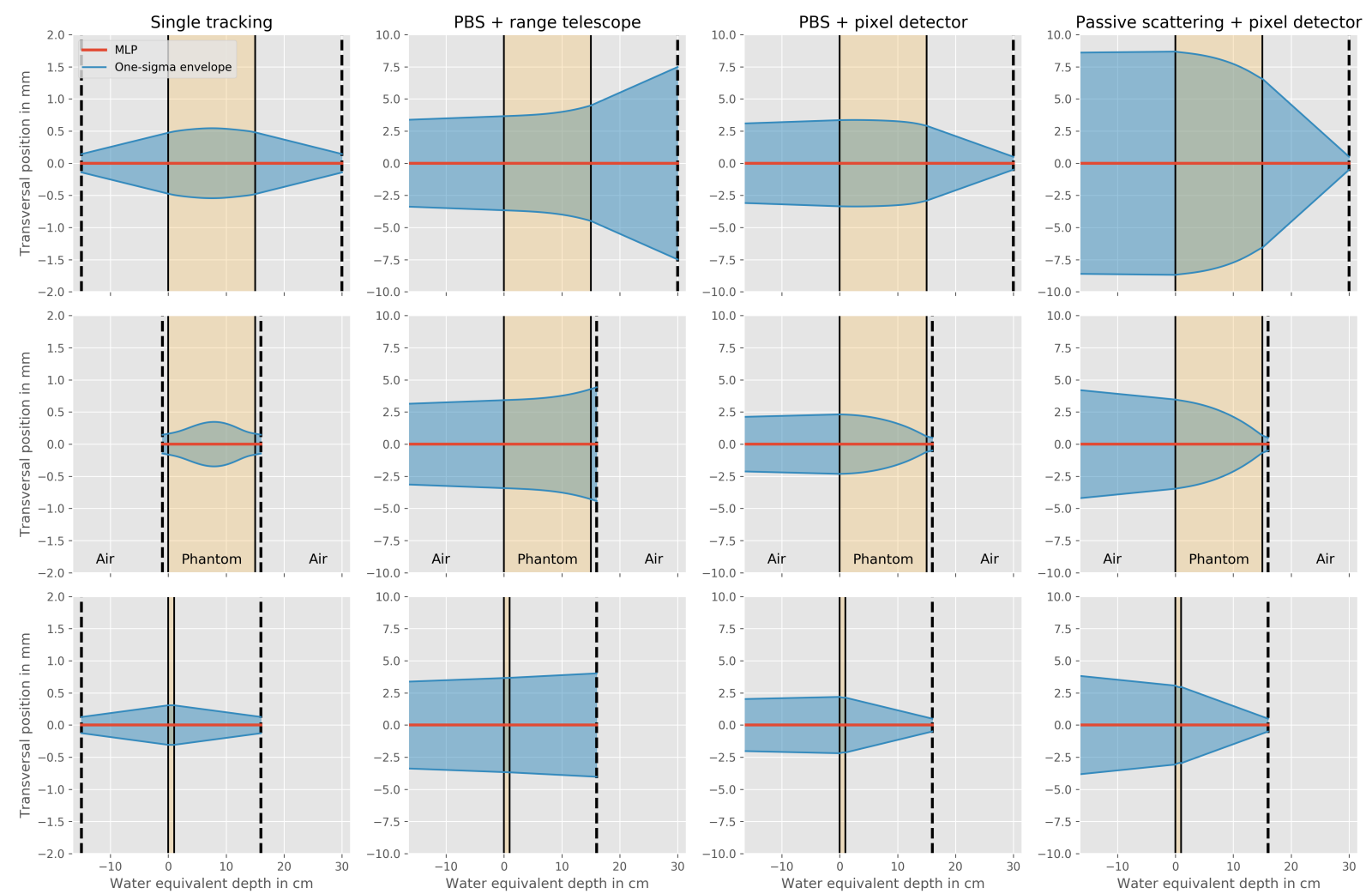

Figure 5: Uncertainty envelopes of the four set-ups calculated using Eq.20 for different phantom thickness values $(1 \mathrm{~cm}$ and $15 \mathrm{~cm}$, respectively) and detector to phantom distances. For the single tracking set-up, the two vertical dashed lines indicate the proton tracker pairs. For the other three set-ups, the vertical dashed line to the right of the phantom refers to the detector. Note the different scale in the leftmost column. 

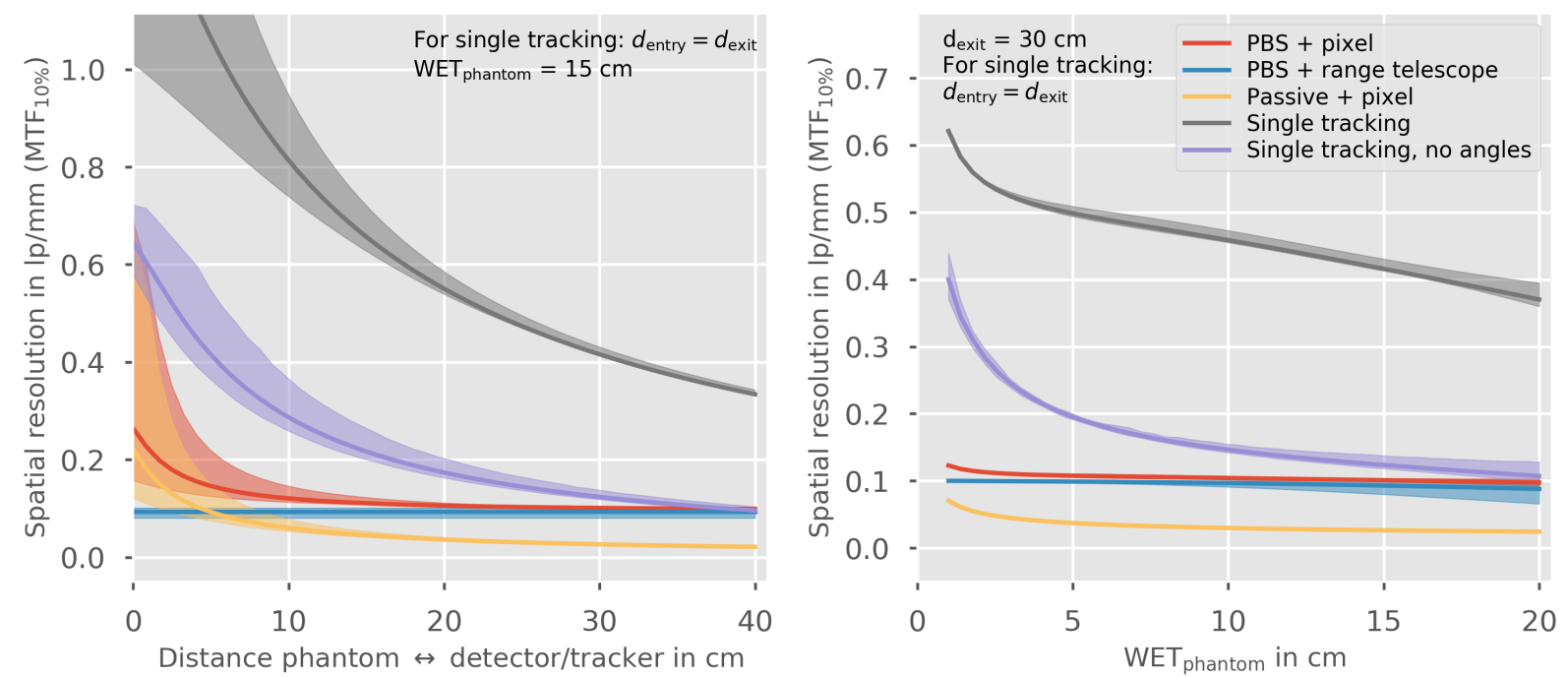

Figure 6: Average spatial resolution $f_{10 \%}^{\text {ave }}$ as a function of detector/tracker distance (left) and phantom thickness (right). The coloured halo depicts the range $\left[f_{10 \%}^{\min }, f_{10 \%}^{\max }\right]$.
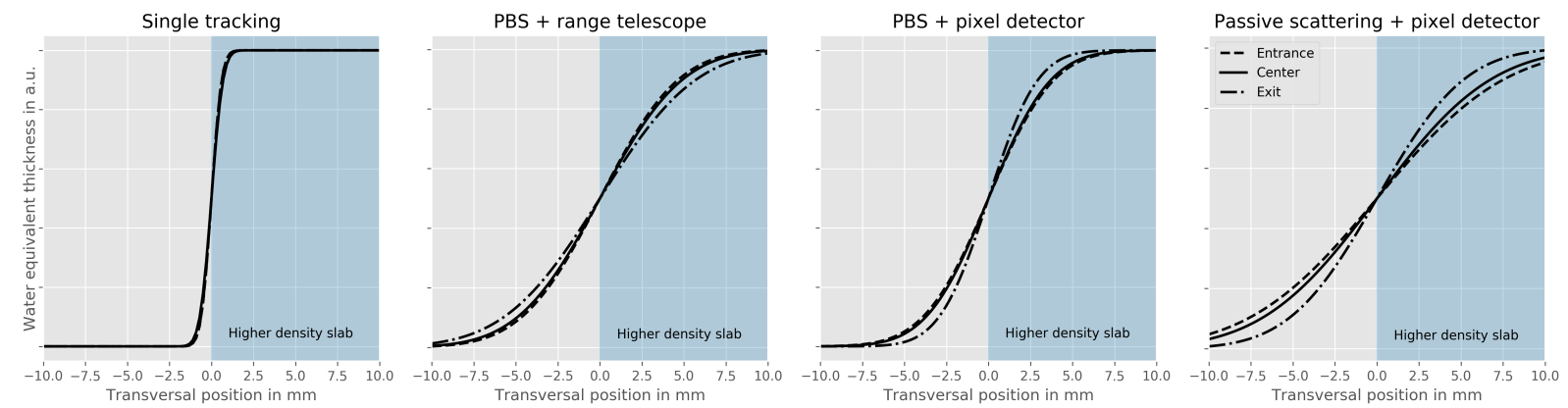

Figure 7: Example of how the back-projected profile of a transversal density step would look like when imaged with the four different set-up types and the geometric parameters as in the first row of Fig. 5. The three lines (dashed, solid, dash-dotted) correspond to the density step positioned in three different depths.
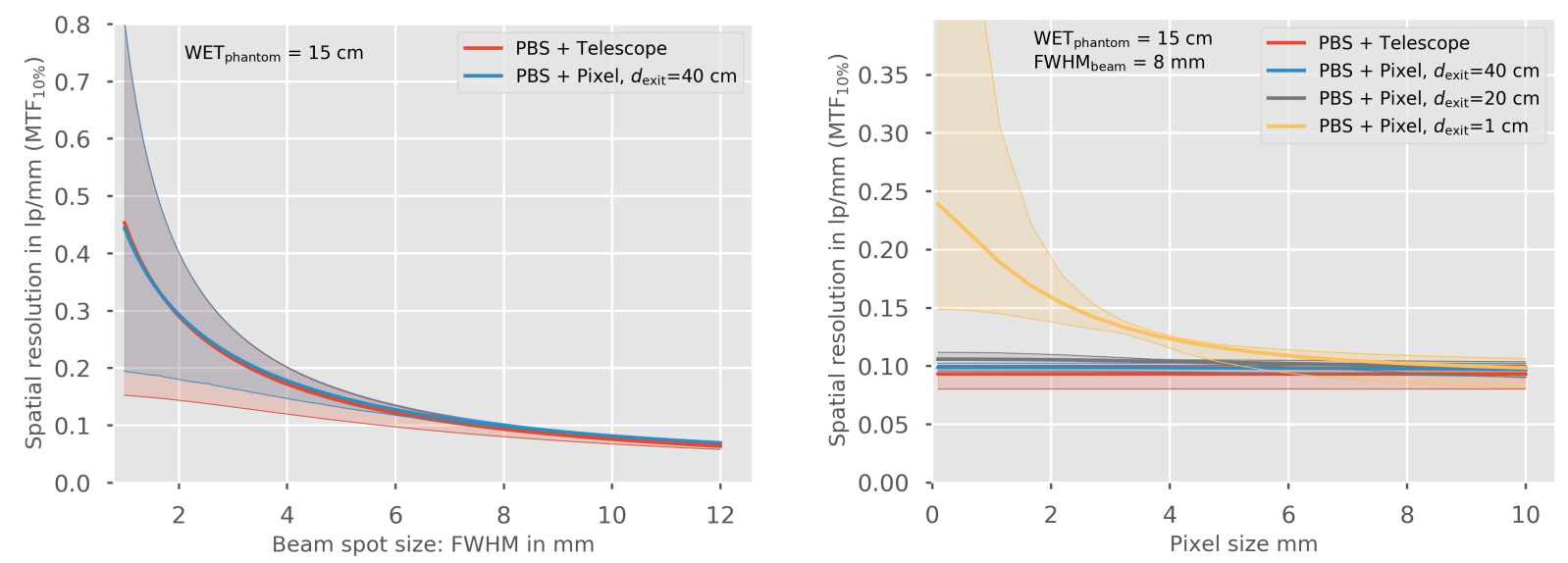

Figure 8: Average spatial resolution $f_{10 \%}^{\text {ave }}$ as a function of beam spot size (left panel) and pixel size (right panel) for PBS + pixel and PBS + range telescope set-ups for $\mathrm{WET}_{\text {phantom }}=15 \mathrm{~cm}$. The coloured halo depicts the range $\left[f_{10 \%}^{\min }, f_{10 \%}^{\max }\right]$. 
Fig. 8 shows the spatial resolution as a function of pixel size in the PBS + pixel set-up for three different distances of the flat panel to the phantom and for a phantom thickness of $15 \mathrm{~cm}$. The PBS + range telescope set-up is shown as reference.

\section{DISCUSSION}

The purpose of this work was to characterise and compare four types of proton imaging set-ups (see Sec. 2.1) under the aspect of spatial resolution. We first verified validity of our analytical formalism by comparing the predicted width of the uncertainty envelope, $\sigma_{\mathrm{MLP}}(u)$, with the values extracted from MC simulations.

The values of $\sigma_{\mathrm{MLP}}(u)$ shown in Fig. 3 agree to within a few percent. We attribute the slight discrepancies partly to the fact that the analytical formalism assumes Gaussian uncertainties/distributions of the entry and exit coordinates while the filtering which we applied in post processing the data corresponds to a uniform (rectangular shaped) distribution. Secondly, our analytical formalism remains within the Gaussian approximation of MCS while Geant4 also simulates larger angle scattering events. Finally, it is known that the MCS models implemented in Geant4 (and other MC codes) as well as experimental data show a few percent variation when compared to each other (Makarova et al. 2017). In this light, our analytical formalism appears to be sufficiently accurate as a means to predict and compare the spatial resolution achievable by the various proton imaging set-ups.

From a mathematical point of view, the spatial resolution is linked to the uncertainty of the projection model: The larger the width of the uncertainty envelope around the estimated projection path, the blurrier the image. As a result, the (back) projected image of a density step oriented transversally to the beam axis would be smeared out, specifically into an error function within the Gaussian approximation of MCS. We used the frequency $f_{10 \%}$ at which the MTF of such an image would decrease below $10 \%$ as a figure of merit for the spatial resolution (see Sec 2.7).

Our formalism calculates the width of the uncertainty envelope in a two dimensional plane, as do all other MLP estimation methods. The full MCS process certainly takes place in three dimensional space and the uncertainty envelope is generally described by a two dimensional depth-dependent distribution around the beam axis. Within the approximation of a homogeneous phantom, this distribution is radially symmetric with $\sigma_{r}=\sqrt{2} \sigma_{\mathrm{MLP}}$.

There is no a priori method to predict the exact spatial resolution in a reconstructed image from the width of the uncertainty envelope. In fact, the spatial resolution in a specific image will depend on the reconstruction algorithm and possibly other image processing techniques such as deconvolution strategies, prior constrained reconstruction, etc. An investigation of such methods was not the purpose of this work. In filtered back projection, however, a direct relation between the size of the uncertainty envelope and the spatial resolution exists: The blurring effect of MCS can be thought of as the result of a Gaussian apodisation function, $A(\nu) \propto$ $\exp \left(-\pi\left(\nu / \nu_{0}\right)^{2}\right)$, filtering out higher frequencies (Panetta \& Demi 2014). The frequency $\nu_{0}$ (in $\mathrm{lp} / \mathrm{mm}$ or cycles $/ \mathrm{mm}$ ) relates to $f_{10 \%}$ as $\nu_{0}=\sqrt{2 \pi / 2 \ln 10} f_{10 \%}$. For a homogeneous, circularly symmetric phantom, the FWHM of the point split function in the center of the reconstructed image will be FWHM $=\sqrt{8 \ln 2} \sigma_{\mathrm{MLP}} \approx 2.35 \sigma_{\mathrm{MLP}}$, where $\sigma_{\mathrm{MLP}}$ is the width of the uncertainty envelope half way into the phantom. More generally, the spatial resolution in a reconstructed tomographic image will depend on the location of the object within the phantom, as observed in other studies (Rit et al. 2013), because the width of the uncertainty envelope depends on depth.

\subsection{Impact of geometric set-up parameters}

With perfect trackers (see Fig. 4), the uncertainty envelope of the single tracking set-up is much thinner than $0.5 \mathrm{~mm}$ at its widest point. The PBS+range telescope set-up shows the expected 
funnel-shaped uncertainty envelope due to MCS. In comparison, in the PBS+pixel detector set-up, the envelope is slightly thinner at the exit surface because the pixels provide additional constraining information on the protons' exit position. This position constraint remains somewhat uncertain despite the infinitely small pixels because of the protons' angular confusion after exiting the phantom and because the angle under which they impinge onto the detector is unknown. The same argument holds for the passive field + pixel detector set-up with the difference that the entrance position of the protons is unknown as they are delivered in a large extended field. For this reason, the uncertainty envelope is very wide $(\approx \pm 8 \mathrm{~mm})$. This is in line with other authors' experimental observation that proton images acquired with this kind of set-up become very blurry once the detector/film is placed several $\mathrm{cm}$ away from the phantom (Seco \& Depauw 2011).

Figs. 5 and 6 report the set-up performance depending on detector distance, detector properties, and phantom thickness. In the single tracking set-up, a resolution of $\approx 1 \mathrm{lp} / \mathrm{mm}$ would be achieved with the trackers placed very close to the phantom. With the trackers placed at $40 \mathrm{~cm}$ distance from the phantom, the limited tracker precision leads to wider uncertainty envelopes towards the phantom surfaces, reaching $1 \mathrm{~mm}$, i.e., $f_{10 \%} \approx 0.3 \mathrm{lp} / \mathrm{mm}$. This is because the angular tracker uncertainty translates into an uncertain entrance position on the phantom surface (mathematically through the matrix $S_{\text {out }}$ in Eq. 15). For realistic distances $(30-40 \mathrm{~cm})$ between the trackers and the patient, the impact of the angular uncertainty on $\sigma_{\mathrm{MLP}}$ and $f_{10 \%}$ therefore tends to be more important than the position uncertainty $\sigma_{p}$ (see Sec. 2.5). Analogous observations have also been reported in (Bopp et al. 2014, Plautz et al. 2016).

For similar reasons, the spatial resolution in the PBS+pixel set-up degrades with increasing detector to phantom distance from $\approx 0.2 \mathrm{lp} / \mathrm{mm}$ to $\approx 0.1 \mathrm{lp} / \mathrm{mm}$. It remains constant $(\approx 0.1 \mathrm{lp} / \mathrm{mm})$ in PBS+range telescope set-up because the detector does anyhow not provide any geometric information.

The performance of single tracking set-ups which do not measure the protons' propagation angle strongly depends on the tracker distance and the phantom thickness. With very thin phantoms $(W E T<2 \mathrm{~cm})$ or when the trackers are placed very close to the phantom $\left(d_{\text {entry }} / d_{\text {exit }}<5 \mathrm{~cm}\right), f_{10 \%}$ decreases only by a factor of two with respect to "full" single tracking set-ups. However, at tracker distances beyond $30 \mathrm{~cm}$ and for phantom thickness of more than $\approx 20 \mathrm{~cm}, f_{10 \%}$ reaches the same level as in integrated mode set-ups, again, because of the lack of angular information in combination with the protons' angular confusion.

Notably, PBS+range telescope set-ups perform similarly well as PBS+pixel set-ups $\left(\bar{\sigma}_{\mathrm{MLP}}\right.$ only $\approx 10 \%$ larger) for detector distances $>20 \mathrm{~cm}$ despite the fact that they do not provide any constraint on the proton exit position. This is also related to the constraint on beam energy when using flat panel detectors (see Sec 2.8): The beam energy has to match the phantom WET while it can be (in theory) arbitrarily high when using a range telescope meaning less scattering.

Passive scattering set-ups perform slightly worse than under idealised conditions because of the additional angular confusion to be taken into account. The pixel information helps to increase the spatial resolution $f_{10 \%}$ from $\approx 0.03 \mathrm{lp} / \mathrm{mm}$ to $\approx 0.2 \mathrm{lp} / \mathrm{mm}$, only when the detector is placed very close to the phantom (little drift in air). This is a potentially unrealistic requirement for clinical implementation.

Our analysis assumes homogeneous material composition as do other MLP formalisms. In a heterogeneous medium, it was observed that unbalanced scattering enhances the contrast along density interfaces (West \& Sherwood 1972, Quiñones et al. 2016, Zhang et al. 2018). While (Zhang et al. 2018) reports experimental findings specifically for the passsive field + pixel detector set-up, heterogeneous materials will likely lead to similar effects also in other types of systems. Integrating this or similar effects in a suitable post-processing/reconstruction algorithm would require more advanced (forward) projection models which take into account material heterogeneities. Exploiting such effects could potentially increase the spatial resolution and deserves further investigation. 

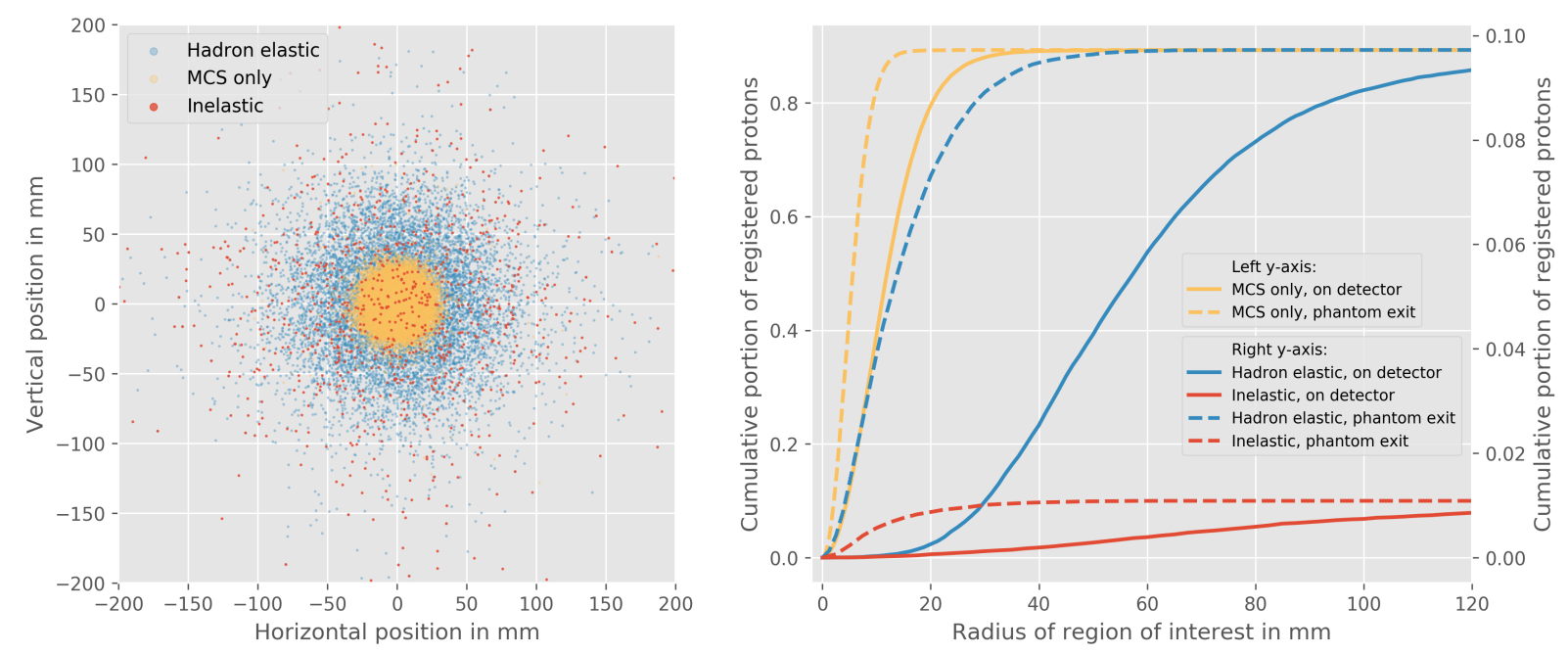

Figure 9: Left: Distribution of protons on the detector surface at $20 \mathrm{~cm}$ distance from the water phantom, colour-coded according to the kind of interaction they have undergone. Right: Relative amount of protons in a circular region of interest of radius $R$ around the beam spot center at the phantom exit surface (dashed) and impinging on the detector surface at $20 \mathrm{~cm}$ distance (solid).

\subsection{Impact of pencil beam spot size and pixel size}

An important result of our study is that the pixel size has an impact on the spatial resolution in PBS + pixel set-ups only if the detector is placed close to the phantom. Beyond a critical distance of about $20 \mathrm{~cm}$ (see Fig. 6, left), the geometrical information of the pixels hardly improves the resolution compared to the PBS + range telescope set-up which does not provide any spatial information at all. The practical implication is that the pixel size does not matter if the detector is placed beyond such a distance. This is explicitly seen in the right panel of Fig. 8 where the spatial resolution remains almost constant except for the yellow curve which refers to a detector distance of $1 \mathrm{~cm}$. The minimum spatial resolution $f_{10 \%}^{\min }$ is lower in the PBS + range telescope set-up because the unconstrained pencil beam widens towards the phantom exit due to MCS.

The main limiting factor for the spatial resolution in the two PBS-based set-ups is the pencil beam spot size (see Fig. 8 left panel). At realistic detector distances $(30-40 \mathrm{~cm})$, the performance of both set-ups is similar. The plot demonstrates that image resolution of both set-ups would benefit significantly from a reduced pencil beam size. A FWHM of $2.5 \mathrm{~mm}$ would, e.g., correspond to a spatial resolution of $\approx 0.25 \mathrm{lp} / \mathrm{mm}$.

Our analytical formalism takes into account the energy loss in an absorber slab to be placed in front of the pixel detector, yet it neglects the additional scattering (see Sec. 2.8). Such scattering "smears out" protons which would have impinged onto the same pixel. Mathematically, the result would be a convolution of a Gaussian kernel describing the additional scattering with the pixel grid. In this sense, the absorber slab increases the effective pixel size of the detector. Since the spatial resolution is largely independent of the pixel size for realistic detector distances, we conclude that neglecting the scattering in the slab does not compromise our results.

\subsection{Region of interest filtering of nuclear interactions}

Nuclear elastic interactions lead to large scattering angles and are the root cause for the wider envelopes obtained with the full physics list (see Fig. 3). The exception is the PBS+pixel setup for which almost no difference in the size of the uncertainty envelope is observed. Protons which have experienced large angle nuclear scattering inside the phantom drift far away from the central beam axis while propagating through air. Therefore, they predominantly impinge onto 
the pixel detector at larger distances from the spot centre than those which have undergone MCS only. This can be seen in the left panel of Fig. 9 where each dot indicates a proton hitting the detector plane at a distance of $20 \mathrm{~cm}$ from the $15 \mathrm{~cm}$ thick phantom. The data were generated using Geant4/Gate with the full physics list (QGSP-BIC). Protons which have undergone at least one nuclear elastic/inelastic interaction along their path are coloured in blue/red, respectively. The right panel shows the cumulative radial distribution for a detector panel positioned immediately at the phantom exit (dashed) and at $20 \mathrm{~cm}$ distance (solid).

This effect can be exploited in PBS + pixel detector set-ups to select protons which have undergone only MCS, similar to what is done in single tracking set-ups in practice where protons which have undergone nuclear interactions are partly filtered out by applying cuts on the exit angle and energy measurements (Schulte et al. 2008). Specifically, using only pixels within a region of interest around the spot centre for a given pencil beam effectively filters out a large portion of nuclear events while retaining almost all protons which have undergone MCS only.

The suitable size of the region of interest depends on the detector distance and on the beam spot size due to MCS and would therefore need to be determined individually for each pencil beam in a real image acquisition. In case of Fig. 9, right panel, the appropriate radius would be about $30 \mathrm{~mm}$. When the region of interest is only a single pixel, as assumed in Fig. 3, virtually no protons having undergone nuclear interaction are considered. This is the reason why no difference is visible between the two simulation results for the PBS + pixel set-up in Fig. 3 .

Because the spatial resolution does anyhow not depend on the pixel size at realistic detector distances (see Fig. 8, right panel), binning adjacent pixels does not compromise the spatial resolution due to MCS (see Sec. 4.2). On the other hand, the here outlined region of interest driven binning strategy might help reduce the impact of nuclear scattering by filtering out most such events.

\subsection{Impact of experimental uncertainties on MLP in single tracking set-ups}

In tomographic reconstruction based on data acquired with a single tracking set-up, the forward projection is performed along the MLP. According to Eq. 21, this depends on the tracker uncertainties, so that the parametrisation of the tracker geometry (see Sec. 2.5p must in general be known and included. In Fig. 10, we show an example MLP calculated for three different tracker uncertainties and for two different phantom thickness values. The exit position and angle were chosen so that $t_{\text {exit }}=\sigma_{t_{\text {exit }}}$ and $\theta_{\text {exit }}=\sigma_{\theta_{\text {exit }}}$ to consider a statistically significant case. With a relatively thin phantom of $5 \mathrm{~cm}$ WET (left panel), the MLP is shifted by about $1 \mathrm{~mm}$ for the less precise tracker compared to the ideal trackers. At the same time, the uncertainty envelope around the MLP is on the same order of magnitude as this systematic difference. The same holds true for the more precise (and more realistic) tracker. With a $20 \mathrm{~cm}$ thick phantom (right panel), the uncertainty due to MCS becomes more dominant than the tracker uncertainty (competing terms in Eq. 21) and the difference between the MLP estimates is smaller than the uncertainty envelope, i.e., essentially insignificant.

This analysis suggests that the tracker uncertainties can potentially be ignored when estimating the MLP using certain parametrisations. Our analytical expressions (Eqs. 21 and 20) provide the mathematical tools to verify this for a specific single tracking set-up.

\section{CONCLUSION}

The purpose of this work was to compare four types of proton imaging set-ups under the aspect of spatial image resolution. We extended the existing formalism of the most likely path to derive analytical expressions which take into account the geometric parameters of the imaging set-up as well as the measurement uncertainties. We considered parameters characteristic for each type of set-up without explicitly referring to any specific implementation reported in the literature. 

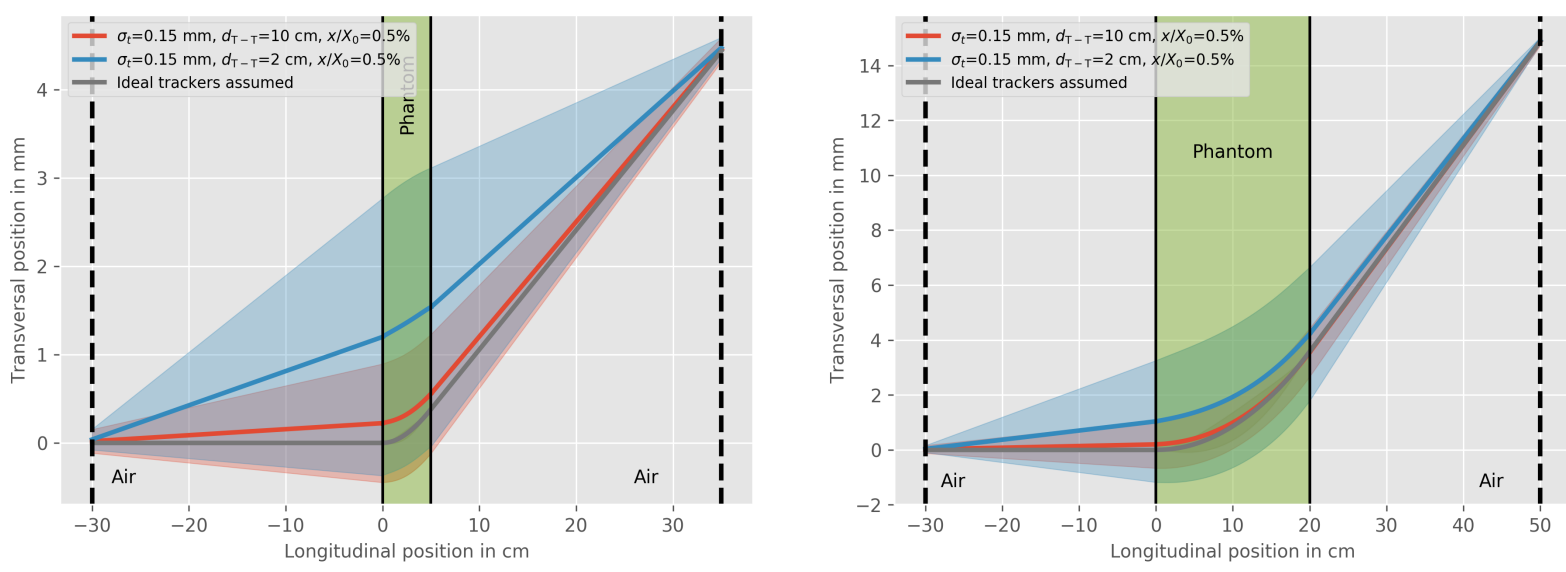

Figure 10: MLP estimates for different tracker characteristics (see Sec. 2.5) and for two different phantom thickness values: $5 \mathrm{~cm}$ (left) and $15 \mathrm{~cm}$ (right).

We analytically calculated the width of the uncertainty envelope around the most likely path, $\bar{\sigma}_{\text {MLP }}$ and used the spatial frequency $f_{10 \%}$ at which the MTF of a transversal density step decays below $10 \%$ as figure of merit for the spatial resolution to compare the set-ups.

Not surprisingly, a single tracking set-up yields the best results in terms of spatial resolution. Our analysis underlined the importance of using tracking detectors with a high angular precision (and accuracy) because an uncertainty in this measurement translates into an uncertain estimate of the entry and exit position on the phantom surface and therefore into an increased uncertainty envelope around the MLP. This effect becomes more important the further away the trackers are placed from the phantom/patient. The distance of the trackers to the patient is largely dictated by the requirement to integrate a proton imaging set-up into a treatment room next to the patient couch and the beam nozzle. In our opinion, $30-40 \mathrm{~cm}$ is actually a realistic value in analogy to the dimensions of current cone beam CT systems. At such a distance, the tracker uncertainty has indeed a larger impact on spatial resolution than multiple scattering within the phantom and we found a spatial resolution of $\approx 0.3-0.35 \mathrm{lp} / \mathrm{mm}$ for typical tracker characteristics.

Our comparison showed that the passive double scattering + pixel detector set-up would be able to produce a satisfactory image resolution only if the detector were very close to the phantom. At $30-40 \mathrm{~cm}$, we found $f_{10 \%}<0.03 \mathrm{lp} / \mathrm{mm}$, so that such a type of imaging set-up will most likely not produce sufficient image resolution for proton CT. Nonetheless, it might be useful in an application in which the detector can be placed very close to the patient, e.g., for range verification measurements ( $\mathrm{Lu} 2008$ ). More advanced post-processing techniques which exploit unbalanced scattering in heterogeneous medium might help improve the spatial resolution (Zhang et al. 2018).

The two set-ups based on pencil beam scanning showed similar results compared with each other $\left(f_{10 \%} \approx 0.1 \mathrm{lp} / \mathrm{mm}\right)$ at realistic detector to phantom distances and for an $8 \mathrm{~mm}$ FWHM pencil beam. The set-up using a position sensitive device brought only a slight advantage in terms of spatial resolution compared to the one using a position insensitive detector. In particular, small pixels do not offer any advantage and we conclude that any pixel size of $1 \mathrm{~mm}$ or less would be sufficient.

An advantage of the pixel detector is that the majority of protons which have undergone large angle nuclear scattering events inside the phantom can be filtered out by using only pixels within a region of interest around the centre of the beam spot. On the other hand, the single plane pixel detector requires multiple irradiations of the phantom/patient with different energies while a single irradiation suffices with a range telescope, or generally a stack of detectors. Which of the two PBS-based set-ups would be preferable in a clinical implementation depends not so much on 
the achievable spatial resolution, but rather factors such as integrability into the treatment room, dosimetric aspects, the time needed for image acquisition, etc. Investigation and comparison of these aspects were not the purpose of this work.

Finally, the spatial resolution achievable with the two PBS-based set-ups is limited mainly as a result of the pencil beam spot size. The resolution could be drastically improved by using very thin pencil beams. For a FWHM of $2.35 \mathrm{~mm}$, we find $f_{10 \%} \approx 0.2-0.25 \mathrm{lp} / \mathrm{mm}$, which is only $50 \%$ worse compared to a single tracking set-up with trackers at $30-40 \mathrm{~cm}$ from the phantom.

\section{Acknowledgements}

The work of Nils Krah was supported partially by a postdoc fellowship of the "German Academic Exchange Service" (DAAD) and partially by funding from the European Union's Horizon 2020 research and innovation programme under the Marie Sklodowska-Curie grant agreement No 753370. Feriel Khellaf was supported by the project DIC20161236452 of the Fondation pour la Recherche Médicale (FRM). The work of Ilaria Rinaldi was supported partially by a research fellowship "Visiting Professor Sapienza" from the University of Rome. This work was performed within the framework of the SIRIC LYriCAN Grant INCa_INSERM_DGOS_12563 and the LABEX PRIMES (ANR-11-LABX-0063) of Université de Lyon, within the program "Investissements d'Avenir" (ANR-11-IDEX-0007) operated by the ANR.

\section{References}

Agostinelli S, Allison J, Amako K, Apostolakis J, Araujo H, Arce P, Asai M, Axen D, Banerjee S, Barrand G, Behner F, Bellagamba L, Boudreau J, Broglia L, Brunengo A, Burkhardt H, Chauvie S, Chuma J, Chytracek R, Cooperman G, Cosmo G, Degtyarenko P, Dell'Acqua A, Depaola G, Dietrich D, Enami R, Feliciello A, Ferguson C, Fesefeldt H, Folger G, Foppiano F, Forti A, Garelli S, Giani S, Giannitrapani R, Gibin D, Gomez Cadenas J J, Gonzalez I, Gracia Abril G, Greeniaus G, Greiner W, Grichine V, Grossheim A, Guatelli S, Gumplinger P, Hamatsu R, Hashimoto K, Hasui H, Heikkinen A, Howard A, Ivanchenko V, Johnson A, Jones F W, Kallenbach J, Kanaya N, Kawabata M, Kawabata Y, Kawaguti M, Kelner S, Kent P, Kimura A, Kodama T, Kokoulin R, Kossov M, Kurashige H, Lamanna E, Lampen T, Lara V, Lefebure V, Lei F, Liendl M, Lockman W, Longo F, Magni S, Maire M, Medernach E, Minamimoto K, Mora de Freitas P, Morita Y, Murakami K, Nagamatu M, Nartallo R, Nieminen P, Nishimura T, Ohtsubo K, Okamura M, O'Neale S, Oohata Y, Paech K, Perl J, Pfeiffer A, Pia M G, Ranjard F, Rybin A, Sadilov S, di Salvo E, Santin G, Sasaki T, Savvas N, Sawada Y, Scherer S, Sei S, Sirotenko V, Smith D, Starkov N, Stoecker H, Sulkimo J, Takahata M, Tanaka S, Tcherniaev E, Safai Tehrani E, Tropeano M, Truscott P, Uno H, Urban L, Urban P, Verderi M, Walkden A, Wander W, Weber H, Wellisch J P, Wenaus T, Williams D C, Wright D, Yamada T, Yoshida H \& Zschiesche D 2003 GEANT4 - A simulation toolkit Nuclear Instruments and Methods in Physics Research, Section A: Accelerators, Spectrometers, Detectors and Associated Equipment 506(3), 250-303.

Amaldi U, Bianchi A, Chang Y H, Go A, Hajdas W, Malakhov N, Samarati J, Sauli F \& Watts D 2011 Construction, test and operation of a proton range radiography system Nuclear Instruments and Methods in Physics Research Section A: Accelerators, Spectrometers, Detectors and Associated Equipment 629(1), 337-344.

URL: http://linkinghub.elsevier.com/retrieve/pii/ S0168900210026318

Arbor N, Dauvergne D, Dedes G, Létang J M, Parodi K, Quiñones C T, Testa E \& Rit S 2015 Monte Carlo comparison of x-ray and proton $\mathrm{CT}$ for range calculations of proton therapy beams Physics in Medicine and Biology 60(19), 7585.

URL: http://stacks.iop.org/0031-9155/60/i=19/a=7585 
Bentefour E H, Schnuerer R \& Lu H M 2016 Concept of proton radiography using energy resolved dose measurement Physics in Medicine and Biology 61(16), N386-N393.

Bopp C, Rescigno R, Rousseau M \& Brasse D 2014 The impact of tracking system properties on the most likely path estimation in protonCT Physics in Medicine and Biology 59(23), N197N210.

Bortfeld T \& Schlegel W 1996 An analytical approximation of depth - dose distributions for therapeutic proton beams Physics in Medicine and Biology 41(8), 1331-1339.

URL: $h t t p: / / s t a c k s . i o p . o r g / 0031-9155 / 41 / i=8 / a=006 ?$ key=crossref.7baffd69782225379bdd466dc31d7b94

Civinini C, Bruzzi M, Bucciolini M, Carpinelli M, Cirrone G A, Cuttone G, Lo Presti D, Pallotta S, Pugliatti C, Randazzo N, Romano F, Scaringella M, Sipala V, Stancampiano C, Talamonti C, Vanzi E \& Zani M 2013 Recent results on the development of a proton computed tomography system Nuclear Instruments and Methods in Physics Research, Section A: Accelerators, Spectrometers, Detectors and Associated Equipment 732, 573-576.

URL: http://dx.doi.org/10.1016/j.nima.2013.05.147

Collins-Fekete C A, Volz L, Portillo S K N, Beaulieu L \& Seco J 2016 A theoretical framework to predict the most likely ion path in particle imaging (October), 1-20.

URL: $h t t p: / / a r x i v . o r g / a b s / 1610.05774$

Coutrakon G, Blazey G, Boi S, Dyshkant A, Erdelyi B, Hedin D, Johnson E, Krider J, Rykalin V, Uzunyan S, Zutshi V, Fordt R, Sellberg G, E. Rauch J, Roman M, Rubinov P, Wilson P \& Naimuddin M 2014 A New Proton CT Scanner 23rd Conference on Application of Accelerators in Research and Industry .

Erdelyi B 2009 A comprehensive study of the most likely path formalism for proton-computed tomography Phys. Med. Biol. Phys. Med. Biol 54(54), 6095-6095.

URL: http://iopscience.iop.org/0031-9155/54/20/005

Farace P, Righetto R \& Meijers A 2016 Pencil beam proton radiography using a multilayer ionization chamber Physics in medicine and biology 61(11), 4078-87.

URL: http://www.ncbi.nlm.nih.gov/pubmed/27164479

Gottschalk B, Koehler A, Schneider R, Sisterson J \& Wagner M 1993 Multiple Coulomb scattering of $160 \mathrm{MeV}$ protons Nuclear Instruments and Methods in Physics Research Section B: Beam Interactions with Materials and Atoms 74(4), 467-490.

URL: http://www.sciencedirect.com/science/article/pii/0168583X9395944Z

Grusell E, Montelius A, Brahme A, Rikner G \& Russell K 1994 A general solution to charged particle beam flattening using an optimized dual-scattering-foil technique, with application to proton therapy beams Physics in Medicine and Biology 39(12), 2201-2216.

Hansen D C, Petersen J B B, Bassler N \& Sørensen T S 2014 Improved proton computed tomography by dual modality image reconstruction. Medical physics 41(3), 031904.

URL: http://www.ncbi.nlm.nih.gov/pubmed/24593722

Hansen D C, Sangild Sørensen T \& Rit S 2016 Fast reconstruction of low dose proton CT by sinogram interpolation Physics in Medicine and Biology 61(15), 5868-5882.

URL: $h$ ttp://stacks.iop.org/0031-9155/61/i=15/a=5868? key=crossref.3d84339bf8b1a 45 e6eae572f6b546

Jan S, Benoit D, Becheva E, Carlier T, Cassol F, Descourt P, Frisson T, Grevillot L, Guigues L, Maigne L, Morel C, Perrot Y, Rehfeld N, Sarrut D, Schaart D R, Stute S, Pietrzyk U, Visvikis D, Zahra N \& Buvat I 2011 GATE V6: A major enhancement of the GATE simulation platform enabling modelling of $\mathrm{CT}$ and radiotherapy Physics in Medicine and Biology 56(4), 881-901. 
Jee K W, Zhang R, Bentefour E H, Doolan P J, Cascio E, Sharp G, Flanz J \& Lu H M 2017 Investigation of time-resolved proton radiography using x-ray flat-panel imaging system Physics in Medicine and Biology 62(5), 1905-1919.

Johnson R P 2018 Review of medical radiography and tomography with proton beams Reports on Progress in Physics 81(1).

Krah N, Testa M, Brons S, Jäkel O, Parodi K, Voss B \& Rinaldi I 2015 An advanced image processing method to improve the spatial resolution of ion radiographies. Physics in medicine and biology 60(21), 8525-8547.

URL: http://iopscience.iop.org/article/10.1088/0031-9155/60/21/8525

Lu H M 2008 A potential method for in vivo range verification in proton therapy treatment. Physics in medicine and biology 53(5), 1413-24.

URL: http://stacks.iop.org/0031-9155/53/i=5/a=016

Lynch G R \& Dahl O I 1991 Approximations to multiple Coulomb scattering Nuclear Inst. and Methods in Physics Research, B 58(1), 6-10.

Makarova A, Gottschalk B \& Sauerwein W 2017 Comparison of Geant4 multiple Coulomb scattering models with theory for radiotherapy protons Physics in Medicine and Biology 62(15), 5959-5974.

Muraishi H, Nishimura K, Abe S, Satoh H, Hara S, Hara H, Takahashi Y, Mogaki T, Kawai R, Yokoyama K, Yasuda N, Tomida T, Ohno Y \& Kanai T 2009 Evaluation of spatial resolution for heavy ion $\mathrm{CT}$ system based on the measurement of residual range distribution with HIMAC IEEE Transactions on Nuclear Science 56(5), 2714-2721.

Paganetti H 2012 a Proton Therapy Physics Taylor \& Francis Group Boca Raton.

Paganetti H $2012 b$ Range uncertainties in proton therapy and the role of Monte Carlo simulations Phys. Med. Biol. 57, R99-R117.

Panetta D \& Demi M 2014 in A Brahme, ed., 'Comprehensive Biomedical Physics' Elsevier Oxford pp. xiii - xvi.

URL: https://www.sciencedirect.com/science/article/pii/ B9780444536327099913

Parodi K 2014 Heavy ion radiography and tomography Physica Medica 30(5), 539-543.

URL: $h t t p: / / d x . d o i . o r g / 10.1016 / j$. ejmp.2014.02.004

Pemler P, Besserer J, de Boer J, Dellert M, Gahn C, Moosburger M, Schneider U, Pedroni E \& Stäuble H 1999 A detector system for proton radiography on the gantry of the Paul-Scherrer-Institute Nuclear Instruments and Methods in Physics Research Section A: Accelerators, Spectrometers, Detectors and Associated Equipment 432(2-3), 483-495.

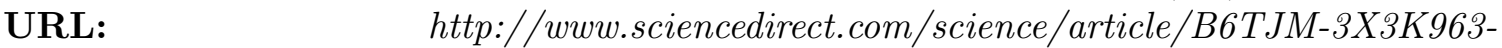
14/2/90849e49134df2c303ea1d298c2f252d

Penfold S N, Rosenfeld A B, Schulte R W \& Sadrozinksi H F 2011 Geometrical optimization of a particle tracking system for proton computed tomography Radiation Measurements 46(12), 2069-2072.

URL: http://dx.doi.org/10.1016/j.radmeas.2011.04.032

Plautz T E, Bashkirov V, Giacometti V, Hurley R F, Johnson R P, Piersimoni P, Sadrozinski H F, Schulte R W \& Zatserklyaniy A 2016 An evaluation of spatial resolution of a prototype proton CT scanner 43(December), 6291-6300.

URL: $h t t p: / / d x . d o i . o r g / 10.1118 / 1.4966028$ 
Poludniowski G, Allinson N M \& Evans P M 2015 Proton radiography and tomography with application to proton therapy British Journal of Radiology 88(1053), 1-14.

Quiñones C T, Létang J M \& Rit S 2016 Filtered back-projection reconstruction for attenuation proton CT along most likely paths Physics in Medicine and Biology 61(9), 3258-3278. URL: http://stacks.iop.org/0031-9155/61/i=9/a=3258? key=crossref.000ce6a57c97a1107276b548832c28

Rescigno R, Bopp C, Rousseau M \& Brasse D 2015 A pencil beam approach to proton computed tomography. Medical physics 42(11), 6610-24.

URL: http://www.ncbi.nlm.nih.gov/pubmed/26520752

Richard S, Carolina N, Husarik D B \& Murphy S N 2012 Towards task-based assessment of CT performance : System and object MTF across different reconstruction algorithms 39(7), 4115-4122.

Rinaldi I, Brons S, Gordon J, Panse R, Voss B, Jäkel O \& Parodi K 2013 Experimental characterization of a prototype detector system for carbon ion radiography and tomography. Physics in medicine and biology 58(3), 413-27.

URL: http://stacks.iop.org/0031-9155/58/i=3/a=413

Rinaldi I, Brons S, Jäkel O, Voss B \& Parodi K 2014 Experimental investigations on carbon ion scanning radiography using a range telescope. Physics in medicine and biology 59(12), 304157.

URL: http://stacks.iop.org/0031-9155/59/i=12/a=3041

Rit S, Dedes G, Freud N, Sarrut D \& Létang J M 2013 Filtered backprojection proton CT reconstruction along most likely paths. Medical physics 40(3), 031103.

URL: http://www.ncbi.nlm.nih.gov/pubmed/23464283

Sarrut D, Bardiès M, Boussion N, Freud N, Jan S, Létang J m, Maigne L, Marcatili S, Mauxion T, Papadimitroulas P, Perrot Y, Pietrzyk U, Robert C, Schaart D R, Visvikis D, Buvat I, Bardiès M, Boussion N, Jan S \& Maigne L 2014 A review of the use and potential of the GATE Monte Carlo simulation code for radiation therapy and dosimetry applications A review of the use and potential of the GATE Monte Carlo simulation code for radiation therapy and dosimetry applications $\mathbf{8 0 9}(2009)$.

Scaringella M, Bruzzi M, Bucciolini M, Carpinelli M, Cirrone G A P, Civinini C, Cuttone G, Lo Presti D, Pallotta S, Pugliatti C, Randazzo N, Romano F, Sipala V, Stancampiano C, Talamonti C, Vanzi E \& Zani M 2014 A proton Computed Tomography based medical imaging system Journal of Instrumentation 12009, 1-8.

Schneider U, Besserer J \& Hartmann M 2012 Technical Note: Spatial resolution of proton tomography: Impact of air gap between patient and detector Medical Physics 39(2), 798800 .

Schneider U, Besserer J, Pemler P, Dellert M, Moosburger M, Pedroni E \& Kaser-Hotz B 2004 First proton radiography of an animal patient. Medical Physics 31(5), 1046-1051.

Schneider U, Pemler P, Besserer J, Pedroni E, Lomax A \& Kaser-Hotz B 2005 Patient specific optimization of the relation between CT-Hounsfield units and proton stopping power with proton radiography Medical Physics 32(1), 195-199.

URL: http://link.aip.org/link/?MPH/32/195/1

Schulte R W, Bashkirov V, Li T, Liang Z, Mueller K, Heimann J, Johnson L R, Keeney B, Sadrozinski H W, Seiden A, Williams D C, Zhang L, Li Z, Peggs S, Satogata T J \& Woody C 2004 Conceptual design of a proton computed tomography system for applications in proton radiation therapy IEEE Trans. Nucl. Sci. 51, 866-872. 
Schulte R W, Bashkirov V, Loss Klock M C, Li T, Wroe A J, Evseev I, Williams D C \& Satogata T 2005 Density resolution of proton computed tomography 32(4), 1035-1046.

URL: http://dx.doi.org/doi/10.1118/1.1884906

Schulte R W \& Penfold S N 2012 Proton CT for Improved Stopping Power Determination in Proton Therapy, invited Trans Am Nucl Soc 106, 55-58.

URL: http://www.ncbi.nlm.nih.gov/pubmed/24771877

Schulte R W, Penfold S N, Tafas J T \& Schubert K E 2008 A maximum likelihood proton path formalism for application in proton computed tomography Medical Physics 35(11), 4849.

URL: http://scitation.aip.org/content/aapm/journal/medphys/35/11/ $10.1118 / 1.2986139$

Seco J \& Depauw N 2011 Proof of principle study of the use of a CMOS active pixel sensor for proton radiography Medical Physics 38(2), 622-623.

Seco J, Oumano M, Depauw N, Dias M F, Teixeira R P \& Spadea M F 2013 Characterizing the modulation transfer function (MTF) of proton/carbon radiography using Monte Carlo simulations. Medical physics 40(9), 091717.

URL: http://www.ncbi.nlm.nih.gov/pubmed/24007150

Shinoda H, Kanai T \& Kohno T 2006 Application of heavy-ion CT Physics in Medicine and Biology 51(16), 4073.

URL: http://stacks.iop.org/0031-9155/51/i=16/a=013

Sipala V, Randazzo N, Aiello S, Bruzzi M, Bucciolini M, Carpinelli M, Cirrone G, Civinini C, Cuttone G, Leonora E, Presti D L, Pallotta S, Pugliatti C, Scaringella M, Stancampiano C, Talamonti C \& Vanzi E 2015 Design and characterisation of a YAG(Ce) calorimeter for proton Computed Tomography application Journal of Instrumentation 10(03), C03014C03014.

URL: $\quad h t t p: / / s t a c k s . i o p . o r g / 1748-0221 / 10 / i=03 / a=C 03014$ ? key=crossref.23aca1811fa4e1953d9e5a7ec304cb68

Taylor J, Allport P, Casse G, Smith N, Tsurin I, Allinson N, Esposito M, Kacperek A, Nieto-Camero J, Price T \& Waltham C 2015 Proton tracking for medical imaging and dosimetry Journal of Instrumentation 10(April 2016), C02015-C02015.

URL: $\quad h t t p: / / s t a c k s . i o p . o r g / 1748-0221 / 10 / i=02 / a=C 02015$ ? key $=$ crossref.9aba03f949b463920287190a3984ccdb

Taylor J T, Poludniowski G, Price T, Waltham C, Allport P P, Casse G L, Esposito M, Evans P M, Green S, Manger S, Manolopoulos S, Nieto-Camero J, Parker D J, Symons J \& Allinson N M 2016 An experimental demonstration of a new type of proton computed tomography using a novel silicon tracking detector Medical Physics 43(11), 6129-6136.

URL: http://doi.wiley.com/10.1118/1.4965809

Telsemeyer J, Jäkel O \& Martisikova M 2012 Quantitative carbon ion beam radiography and tomography with a flat-panel detector Phys. Med. Biol. 57, 7957-7971.

Testa M, Verburg J M, Rose M, Min C H, Tang S, Bentefour E H, Paganetti H \& Lu H M 2013 Proton radiography and proton computed tomography based on time-resolved dose measurements Physics in Medicine and Biology 58(22), 8215.

URL: http://stacks.iop.org/0031-9155/58/i=22/a=8215

West D \& Sherwood A 1972 Radiography with $160 \mathrm{MeV}$ protons Nature 239, 157-159.

URL: http://www.nature.com/nature/journal/v239/n5368/abs/239157b0.html 
Williams D C 2004 The most likely path of an energetic charged particle through a uniform medium Physics in Medicine and Biology 49(13), 2899-2911.

URL: http://stacks.iop.org/0031-9155/49/i=13/a=010

Zhang R, Jee K W, Cascio E, Sharp G C, Flanz J B \& Lu H M 2018 Improvement of single detector proton radiography by incorporating intensity of time-resolved dose rate functions Physics in Medicine 85 Biology 63(1), 15030.

URL: http://stacks.iop.org/0031-9155/63/i=1/a=015030

Zygmanski P, Gall K P, Rabin M S \& Rosenthal S J 2000 The measurement of proton stopping power using proton-cone-beam computed tomography. Physics in medicine and biology 45(2), 511-28.

URL: http://www.ncbi.nlm.nih.gov/pubmed/10701518 